\title{
On the formation of terrestrial planets in hot-Jupiter systems
}

\author{
M. J. Fogg and R. P. Nelson
}

\author{
Astronomy Unit, Queen Mary, University of London, Mile End Road, London E1 4NS, UK \\ e-mail: [M.J.Fogg; R.P.Nelson]@qmul.ac.uk
}

Received 3 August 2006 / Accepted 9 October 2006

ABSTRACT

\begin{abstract}
Context. There are numerous extrasolar giant planets which orbit close to their central stars. These "hot-Jupiters" probably formed in the outer, cooler regions of their protoplanetary disks, and migrated inward to $\sim 0.1 \mathrm{AU}$. Since these giant planets must have migrated through their inner systems at an early time, it is uncertain whether they could have formed or retained terrestrial planets.

Aims. We present a series of calculations aimed at examining how an inner system of planetesimals/protoplanets, undergoing terrestrial planet formation, evolves under the influence of a giant planet undergoing inward type II migration through the region bounded between 5-0.1 AU.

Methods. We have previously simulated the effect of gas giant planet migration on an inner system protoplanet/planetesimal disk using a $N$-body code which included gas drag and a prescribed migration rate. We update our calculations here with an improved model that incorporates a viscously evolving gas disk, annular gap and inner-cavity formation due to the gravitational field of the giant planet, and self-consistent evolution of the giant's orbit.

Results. We find that $\gtrsim 60 \%$ of the solids disk survives by being scattered by the giant planet into external orbits. Planetesimals are scattered outward almost as efficiently as protoplanets, resulting in the regeneration of a solids disk where dynamical friction is strong and terrestrial planet formation is able to resume. A simulation that was extended for a few Myr after the migration of the giant planet halted at $0.1 \mathrm{AU}$, resulted in an apparently stable planet of $\sim 2 m_{\oplus}$ forming in the habitable zone. Migration-induced mixing of volatile-rich material from beyond the "snowline" into the inner disk regions means that terrestrial planets that form there are likely to be water-rich.

Conclusions. We predict that hot-Jupiter systems are likely to harbor water-abundant terrestrial planets in their habitable zones. These planets may be detected by future planet search missions.
\end{abstract}

Key words. planets and satellites: formation - methods: $N$-body simulations - astrobiology

\section{Introduction}

About one quarter of the extrasolar planetary systems discovered to date contain a so-called "hot-Jupiter" - a gas giant planet orbiting within $0.1 \mathrm{AU}$ of the central star (e.g. Butler et al. 2006). It is improbable that these planets formed within such a hot region of the protoplanetary disk, and is most likely that they originated further out beyond the nebula snowline and moved inward via type II migration (e.g Lin \& Papaloizou 1986; Ward 1997; Bryden et al. 1999; Nelson et al. 2000). Since both giant planet formation and type II migration require the nebular gas to still be present, these processes are constrained to occur within the first few million years of the disk lifetime (Haisch et al. 2001), well within the $\sim 30-100 \mathrm{Myr}$ timescale thought to be required to complete the growth of terrestrial planets (e.g. Chambers 2001; Kleine et al. 2002; Halliday 2004). Thus, these migrating giant planets must have traversed the inner system, including its habitable zone, before any planet formation there was complete, raising the question of what effect such a disturbance would have on the growth of terrestrial-like planets.

Initially, it was thought reasonable that the inward migration of a giant planet would be so disruptive of the material it passed through as to clear the swept zone completely, precluding the formation of any inner system planets. However, this view was a conservative assumption, often made in the support of speculative astrobiological arguments (e.g. Ward \& Brownlee 2000; Lineweaver 2001; Lineweaver et al. 2004). Agreement as to the outcome failed to materialize from the first modeling studies of the process, the conclusions of which varied from the occurrence of terrestrial planets in hot-Jupiter systems being highly unlikely (Armitage 2003), through possible but rare (Mandell \& Sigurdsson 2003), to commonplace (Raymond et al. 2005a). None of these studies, however, actually simulated terrestrial planet formation simultaneously with giant planet migration. Their disagreement about the likely outcome can be traced to assumptions made about the timing of giant planet formation and migration.

The first study to model inner system planetary accretion in the presence of a migrating giant was that of Fogg \& Nelson (2005) (hereafter referred to as Paper I). This work used $N$-body simulations to examine oligarchic and giant-impact growth (Kokubo \& Ida 2000) in a protoplanet/planetesimal disk based on the minimum mass solar nebula model of Hayashi (1981), extending between 0.4-4 AU. Five scenarios were considered, corresponding to five different ages for the inner planet forming disk at the point when a giant planet was assumed to form at $5 \mathrm{AU}$ and migrate in to $0.1 \mathrm{AU}$.

In all five of their scenarios, Fogg \& Nelson (2005) found that the majority of the disk solids survived the passage of the giant planet, either by being shepherded inward of the giant, or by being scattered by the giant into excited exterior orbits. This partition of solid material was shown to vary with the level of dissipative forces present, which decline with disk maturity, favoring shepherding at early times and scattering at late times. Within the portion of the disk compacted inside the increasingly 
restricted volume interior to the giant, accretion was found to speed up greatly at late times resulting typically in a $\sim 3-15 M_{\oplus}$ planet forming inside $0.1 \mathrm{AU}$. The similarity of these objects to the recently identified class of "hot-Neptune" planets (McArthur et al. 2004; Butler et al. 2004; Santos et al. 2004b; Vogt et al. 2005; Rivera et al. 2005; Bonfils et al. 2005; Udry et al. 2006) was noted and discussed. The fate of the material scattered into external orbits was not subjected to further calculation, but it was noted that, although the remaining solids surface density was reduced from pre-migration-episode values, ample material remained to provide for the eventual accretion of a set of external terrestrial planets, including within each system's habitable zone. Fogg \& Nelson (2005) therefore concluded that the assumption that hot-Jupiter systems are devoid of inner system terrestrial planets is probably incorrect, and that planet formation and retention both interior and exterior to the hot-Jupiter is possible. We note that similar results, relating to the formation of planets interior to a migrating giant, have been reported by Zhou et al. (2005).

In this paper, we extend the model introduced in Paper I by improving the realism with which gas dynamics is simulated. A $1 \mathrm{D}$ evolving viscous gas disk model is linked to the $N$-body code that: 1) allows the gas to deplete over time via viscous accretion onto the central star; 2) allows an annular gap to form in the vicinity of the giant planet; 3 ) includes the creation of an inner cavity due to dissipation of propagating spiral waves excited by the giant planet; 4) self-consistently drives the giant inward. Compared to the unevolving gas disk assumed in Paper I, this new model reduces the strength of dissipation present in all scenarios, especially in regions close to the central star and the giant. We examine and re-interpret the fate of the disk solids under these changed circumstances and look at whether our hypothesis of hot-Neptune formation remains robust. We also examine the post-migration evolution of the outer scattered disk of solids, and find that terrestrial planets do form in the habitable zone. Another issue we examine is the extent to which volatilerich matter, originating from beyond the snowline, is driven into the inner system and is mixed into the surviving planet-forming material. We find that substantial mixing occurs, such that any terrestrial planets that form are likely to be water-rich bodies hosting deep, global oceans.

The plan of the paper is as follows. In Sect. 2 we outline the additions to our model and the initial conditions of the simulations; in Sect. 3 the results are presented and discussed; in Sect. 4 we consider some caveats and future model improvements, and in Sect. 5 we offer our conclusions.

\section{Description of the model}

We model planetary accretion using the Mercury 6 hybridsymplectic integrator (Chambers 1999), run as an $N+N^{\prime}$ simulation, where we have $N$ protoplanets embedded in a disk of $N^{\prime}$ "super-planetesimals" - particles that represent an idealized ensemble of a much larger number of real planetesimals. The protoplanets (and the giant when it is introduced) interact gravitationally and can accrete with all the other bodies in the simulation, whereas the super-planetesimal population is non-selfinteracting. These latter objects however are subject to a drag force from their motion relative to the nebular gas. A detailed outline of these aspects of our model is given in Sects. 2.1 and 2.2 of Paper I and we continue here to discuss the additional features we have introduced.

\subsection{Improved nebula model}

In Paper I we assumed a steady state gas disk model with a constant surface density profile $\propto r^{-1.5}$. The migration rate of the giant was prescribed from a calculation of the local viscous evolution timescale. More realistically, the quantity of nebular gas should decline due to viscous evolution and accretion onto the central star, progressively depleting the inner disk. The gas density should also decrease in the vicinity of the giant due to the generation and dissipation of density waves at Lindblad resonance positions, clearing an annular gap in a zone where the planetary tidal torques dominate the intrinsic viscous torques of the disk. A consistent calculation of the type II migration rate should involve the back-reaction to these tidal torques.

To account for these processes we model the gas disk by solving numerically the disk viscous diffusion equation (Pringle 1981), with modifications included to account for the tidal influence of an embedded giant planet. Such a method has been used previously in studies that attempt to explain the statistical distribution of exoplanetary orbits through type II migration and disk dispersal (Trilling et al. 1998; Armitage et al. 2002; Alibert et al. 2005). The simplest technique for including the effect of the planet is the impulse approximation of Lin \& Papaloizou (1986), where wave dissipation is assumed to occur close to the planet. A more sophisticated treatment of the problem is the WKB approximation (Takeuchi et al. 1996) which involves summing the torque contributions from a series of Lindblad resonances in the disk. The former technique was adopted in the studies cited above as it requires considerably less computation and generates comparable results. We follow this approach here, but in order to include the effect of non-local dissipation of waves that travel far into the disk we also include the WKB approximation torques due to the waves launched at the two innermost and outermost Lindblad resonances.

We assume a MMSN-type protoplanetary disk around a $M_{*}=1 M_{\odot}$ star (Hayashi 1981). The initial surface density of solids is:

$\Sigma_{\mathrm{s}}(r)=f_{\mathrm{neb}} f_{\mathrm{ice}} \Sigma_{1}\left(\frac{r}{1 \mathrm{AU}}\right)^{-1.5}$

where $r$ is radial distance from the central star, $f_{\text {neb }}$ is a nebular mass scaling factor, $\Sigma_{1}=7 \mathrm{~g} \mathrm{~cm}^{-2}$ and the ice condensation coefficient $f_{\text {ice }}=1$ for $a<2.7 \mathrm{AU}$ (the distance chosen for the nebula "snowline") and $f_{\text {ice }}=4.2$ for $a \geq 2.7$ AU. As in Paper I, we set $f_{\text {neb }}=3$.

The initial surface density of gas is:

$\Sigma_{\mathrm{g}}(r)=f_{\mathrm{neb}} f_{\mathrm{gas}} \Sigma_{1}\left(\frac{r}{1 \mathrm{AU}}\right)^{-1.5}$,

where $f_{\text {gas }}$ is the gas to dust ratio which we take to be $f_{\text {gas }}=240$. Given a nebular radial temperature profile of $T_{\text {neb }}=$ $280(r / \mathrm{AU})^{-1 / 2}$, the sound speed of a solar composition gas in cgs units is:

$c_{\mathrm{s}}=9.9 \times 10^{4}\left(\frac{T_{\mathrm{neb}}}{280 \mathrm{~K}}\right)^{\frac{1}{2}}$,

and the gas scale height is:

$h=0.047\left(\frac{r}{1 \mathrm{AU}}\right)^{\frac{5}{4}} \mathrm{AU}$.

Since the kinematic viscosity in an alpha-disk model is $v=$ $\alpha h^{2} \Omega$, where $\Omega$ is the local Keplerian angular velocity, we take the turbulent shear viscosity function of the disk to be:

$v=9.84 \times 10^{16} \alpha\left(\frac{r}{1 \mathrm{AU}}\right)$, 
and the viscous evolution time:

$\tau_{v}=\frac{2}{3}\left(\frac{r}{h}\right)^{2}(\alpha \Omega)^{-1} \approx 10^{-4} \alpha^{-1} r \approx 48 \alpha^{-1}\left(\frac{r}{1 \mathrm{AU}}\right) \mathrm{yr}$.

In all the models presented here we assume a disk alpha viscosity of $\alpha=2 \times 10^{-3}$, giving $\tau_{v}(5 \mathrm{AU}) \approx 120000$ years.

We solve the diffusion equation for $\Sigma_{\mathrm{g}}(r)$ in the form:

$\frac{\partial \Sigma_{\mathrm{g}}}{\partial t}=\frac{1}{r} \frac{\partial}{\partial r}\left[3 r^{\frac{1}{2}} \frac{\partial}{\partial r}\left(v \Sigma_{\mathrm{g}} r^{\frac{1}{2}}\right)-\frac{2 \Lambda \Sigma_{\mathrm{g}} r^{\frac{3}{2}}}{\left(G M_{*}\right)^{\frac{1}{2}}}-\frac{T r^{\frac{1}{2}}}{3 \pi\left(G M_{*}\right)^{\frac{1}{2}}}\right]$,

where the first term in square brackets describes the diffusion of gas under the action of internal viscous torques (Pringle 1981); the second term describes the impulse approximation of the local tidal interaction of the planet with the disk, with $\Lambda$ being the specific torque exerted by the planet (Lin \& Papaloizou 1986); and the third term, which derives from the WKB approximation (Takeuchi et al. 1996), is included to account for more distant angular momentum transfer via the damping of waves launched from the innermost and outermost two Lindblad resonances, with $T$ being a summation of the torque densities exerted by these waves. Because their launch sites stand off a substantial distance from the planet, these waves are expected to be linear and not damped locally in the disk. Their angular momentum content is therefore deposited in the disk through viscous damping as they propagate.

The exchange of angular momentum between the planet and disk leads to a radial migration of the planet at a rate:

$\frac{\mathrm{d} a}{\mathrm{~d} t}=-\left(\frac{a}{G M_{*}}\right)^{\frac{1}{2}} \frac{1}{m_{\mathrm{p}}}\left[4 \pi \int_{r_{\text {in }}}^{r_{\text {out }}} r \Lambda \Sigma_{\mathrm{g}} \mathrm{d} r+2 \int_{r_{\text {in }}}^{r_{\text {out }}} T \mathrm{~d} r\right]$,

where $m_{\mathrm{p}}$ is the mass of the planet, $a$ is its semi-major axis and $r_{\text {in }}$ and $r_{\text {out }}$ are the inner and outer boundaries of the disk respectively.

The rate of specific angular momentum transfer to the disk in the impulse approximation is given by Lin \& Papaloizou (1986) as:

$\Lambda=\operatorname{sign}(r-a) q^{2} \frac{G M_{*}}{2 r}\left(\frac{r}{\left|\Delta_{\mathrm{p}}\right|}\right)^{4}$,

where $q=m_{\mathrm{p}} / M_{*}$, and $\Delta_{\mathrm{p}}=\max (h,|r-a|)$.

The total torque density exerted on the disk in the WKB approximation via the damping of waves excited by the planet is (Takeuchi et al. 1996):

$T(r)=\sum_{m} T_{m}(r)$

where $m$ is the mode number of the $m$ th order Lindblad resonance at:

$r_{\mathrm{L}}=\left(1 \mp \frac{1}{m}\right)^{\frac{2}{3}} a$

where use of the minus sign gives the radial distances of the inner resonances $r_{\mathrm{IL}}$ and the plus sign those of the outer resonances $r_{\mathrm{OL}}$. Since only the innermost and outermost two Lindblad resonances are accounted for here, we take $m=2,3$ for the resonance positions interior and $m=1,2$ for those exterior to the planet.

The torque density is calculated from the radial gradient of the angular momentum flux $F_{m}(r)$ :

$T_{m}(r)=-\frac{\mathrm{d} F_{m}(r)}{\mathrm{d} r}$ which is given in Takeuchi et al. (1996) as:

$$
\begin{aligned}
F_{m}(r)= & F_{m 0} \exp \left[-\int_{r_{\mathrm{L}}}^{r}\left\{\zeta+\left(\frac{4}{3}+\frac{\kappa^{2}}{m^{2}\left(\Omega-\Omega_{\mathrm{p}}\right)^{2}}\right) \nu\right\}\right. \\
& \left.\times \frac{m\left(\Omega_{\mathrm{p}}-\Omega\right)}{c_{\mathrm{s}}^{2}} k \mathrm{~d} \tilde{r}\right],
\end{aligned}
$$

where $F_{m}(r)=0$ for $r_{\mathrm{IL}}<r<r_{\mathrm{OL}}, \zeta$ is the bulk viscosity (set here to zero), $\kappa=\Omega$ for a Keplerian disk, $\Omega_{\mathrm{p}}$ is the angular velocity of the planet and $k(r)$ is the radial wavenumber:

$k(r)=\left[\frac{m^{2}\left(\Omega-\Omega_{\mathrm{p}}\right)^{2}-\kappa^{2}}{c_{\mathrm{s}}^{2}}\right]^{\frac{1}{2}}$.

Takeuchi et al. (1996) give this approximation for the angular momentum flux originating at a given $m$ th order resonance:

$$
\begin{aligned}
F_{m 0}= & \frac{4}{3} m^{2} f_{\mathrm{c}} \Sigma_{\mathrm{g}}\left(r_{\mathrm{L}}\right)\left(\frac{G m_{\mathrm{p}}}{a \Omega_{\mathrm{p}}}\right)^{2} \\
& \times\left[2 \mathrm{~K}_{0}\left(\frac{2}{3}\right)+\mathrm{K}_{1}\left(\frac{2}{3}\right)-\frac{\pi}{2} \delta_{m, 1}(2 \pm 1)\right]^{2},
\end{aligned}
$$

where $\mathrm{K}_{0}$ and $\mathrm{K}_{1}$ are modified Bessel functions, $\delta_{m, 1}$ is the Kronecker delta function, and the upper component of the \pm is used for the inner and the lower component for the outer resonances respectively.

The parameter $f_{\mathrm{c}}$ is a torque cutoff function (Artymowicz 1993) given by:

$f_{\mathrm{c}}=\frac{1}{H\left(1+4 \xi^{2}\right)}\left[\frac{2 H \mathrm{~K}_{0}(2 H / 3)+\mathrm{K}_{1}(2 H / 3)}{2 \mathrm{~K}_{0}(2 / 3)+\mathrm{K}_{1}(2 / 3)}\right]^{2}$,

where $H=\left(1+\xi^{2}\right)^{1 / 2}$ and $\xi=m\left(c_{\mathrm{s}} / a \Omega_{\mathrm{p}}\right)$.

The evolution of the nebular gas is computed by solving Eq. (7) with an explicit finite-difference technique on a grid with a cell width $\Delta r \propto \sqrt{r}$. The resulting type II migration forces on the giant planet are computed from Eq. (8) by deriving an instantaneous time scale, $a / \dot{a}$, and inserting this into Eq. (6) of Paper I. Strong eccentricity damping for the giant planet is assumed, with the damping time scale being 1/50th of the radial migration timescale. As in Paper I, we have neglected the effects of type I migration and associated eccentricity damping (Ward 1997; Tanaka et al. 2002; Tanaka \& Ward 2004).

The gas disk adopted here extends from an inner radius $r_{\text {in }}=0.025 \mathrm{AU}$ to an outer radius of $r_{\text {out }}=33 \mathrm{AU}$ with an initial $\Sigma_{\mathrm{g}}(r)$ profile given by Eq. (2). Since we are considering a $3 \times$ MMSN disk, $f_{\text {neb }}=3$ and the initial disk mass is $M_{\text {gas }}=0.0398 M_{\odot} \approx 42 M_{\mathrm{J}}$. Note that this initial disk mass is greater than the $2 \times$ MMSN of gas assumed in Paper I; however since that amount was kept fixed, the gas present in the simulations presented here falls below this level after $\sim 140000$ years. The boundary conditions for the computation were $\Sigma_{\mathrm{g}}\left(r_{\mathrm{in}}\right)=0$, representing gas accretion onto the central star and at $r_{\text {out }}$ the radial velocity of the gas was set to zero.

To correctly couple the evolving gas disk algorithm with the $\mathrm{N}$-body code, synchronization of their respective time-steps is necessary. In each simulation sub-run (see Sect. 2.3) the symplectic $N$-body time-step $\tau_{\text {nbody }}$ was fixed whereas the gas disk time-step $\tau_{\text {gas }}$ is adaptive and taken to be:

$\tau_{\mathrm{gas}}=\min \left(\frac{w \Delta r(i)}{\left|v_{r}(i)\right|}\right)$ 
where $i$ is the grid cell label and $w$ is a coefficient of order unity that is tuned to ensure computational stability. Including all the torques given above, the gas radial velocity is:

$v_{r}=2 \sqrt{\frac{r}{G M_{*}}}\left(\Lambda+\frac{T}{\Sigma_{\mathrm{g}} r}\right)-\frac{3}{\Sigma_{\mathrm{g}} r^{1 / 2}} \frac{\partial}{\partial r}\left[v \Sigma_{\mathrm{g}} r^{1 / 2}\right]$.

Thus, if $\tau_{\text {gas }} \geqslant \tau_{\text {nbody }}$ then we set $\tau_{\text {gas }}=\tau_{\text {nbody }}$; if $\tau_{\text {gas }}<\tau_{\text {nbody }}$ then the gas disk is evolved for $\operatorname{INT}\left(\tau_{\text {nbody }} / \tau_{\text {gas }}\right)$ steps of duration $\tau_{\text {gas }}$ plus an extra step of $\operatorname{MOD}\left(\tau_{\text {nbody }} / \tau_{\text {gas }}\right)$.

\subsection{Radial mixing of solid material}

At early times, the solid component of a young protoplanetary nebula will exhibit a radial pattern of chemical composition, controlled by the temperature-dependent condensation sequence of a variety of metals, rock minerals and ices. As the planetary system grows and evolves, phenomena such as dynamical spreading, gas drag induced orbital decay and resonant interactions can cause a radial mixing of material. According to one school of thought (e.g. Morbidelli et al. 2000) the original matter that condensed in the Earth's orbit is thought to have been dry (for an alternative opinion see Drake \& Righter 2002) and the origin of the Earth's water, and its $\mathrm{D} / \mathrm{H}$ ratio, can be explained if $\sim 10 \%$ of the planet's mass is composed of carbonaceous chondrite-type material, originating from between 2.5-4 AU, and $\sim 10 \%$ of the water gained thereby is retained at the end of accretion. $N$-body simulations of terrestrial planet formation from disks that extend out close to the orbit of Jupiter are supportive of this idea and all demonstrate substantial mixing of water rich material into the inner disk (e.g. Morbidelli et al. 2000; Chambers 2001; Raymond et al. 2004, 2005a,b).

In Paper I it was noted that one consequence of the inward migration of a giant planet is the shepherding of planetesimals that are damped by gas drag (Tanaka \& Ida 1999), and the trapping of bodies at first order mean motion resonances. Hence the outer, more volatile-rich, regions of the protoplanetary disk are actively mixed into its inner regions. However, the composition of planetesimals and protoplanets, and their accretion products, were not logged in our simulations.

We amend this here by labeling all particles with a composition based on its original position in the disk, and summing the composition of protoplanets as they grow. In a similar manner to most other studies, we assume a crude three-phase initial radial composition with rocky material originating at $<2 \mathrm{AU}$, material characteristic of chondritic meteorites between 2-2.7 AU, and trans-snowline material at $>2.7 \mathrm{AU}$. For convenience, these phases are referred to as "dry", "damp" and "wet" respectively but, in contrast to other studies, we do not assign an actual water mass fraction to them. Instead we use our results to make more generalized observations and predictions.

\subsection{Initial conditions and running of the simulations}

In common with Paper I, we assume a nominal age for the protoplanetary disk of $0.5 \mathrm{Myr}$, this being the $t=0$ start date for our simulations, and a mass of three times that of the MMSN. Our reasons for choosing a more massive nebula stem from the fact that core-accretion theories of giant planet formation require an enhanced density of solid material in order to grow a critical core mass before the nebular gas is lost (Lissauer 1987; Thommes et al. 2003) which might be supported by the observation that hot-Jupiters are found predominantly around stars more metalrich than the Sun (Santos et al. 2004a; Fischer \& Valenti 2005).
Table 1. Data describing initial solids disk set-up.

\begin{tabular}{cccc}
\hline \hline & Rocky zone & Icy zone & Total \\
& $0.4-2.7 \mathrm{AU}$ & $2.7-4.0 \mathrm{AU}$ & $0.4-4.0 \mathrm{AU}$ \\
\hline$M_{\text {solid }}$ & $9.99 M_{\oplus}$ & $14.8 M_{\oplus}$ & $24.8 M_{\oplus}$ \\
$m_{\text {proto }}$ & $0.025 M_{\oplus}$ & $0.1 M_{\oplus}$ & \\
$N$ & 66 & 9 & 75 \\
$m_{\text {s-pl }}$ & $0.0025 M_{\oplus}$ & $0.01 M_{\oplus}$ & \\
$N^{\prime}$ & 3336 & 1392 & 4278 \\
$f_{\text {proto }}$ & 0.17 & 0.06 & 0.1 \\
\hline
\end{tabular}

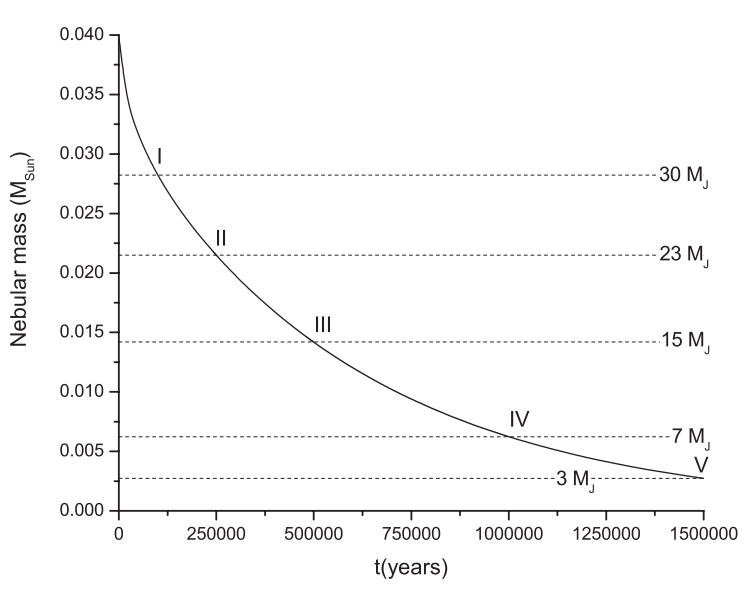

Fig. 1. Evolution of the mass of the gas disk. The mass of gas (in Jupiter masses) remaining at the launch point for each of the five migration scenarios is indicated.

As runs proceed, the initial gas disk described by Eq. (2) evolves according to the algorithm outlined in Sect. 2.1, initially without the presence of a giant planet (i.e. $\Lambda=0, T=0$ in Eq. (7)).

Simultaneously, Mercury 6 evolves an initial disk of solid material, extending radially from 0.4-4.0 AU. We use the same two-component initial solids disk as was used in Paper I, generated according to the profile of Eq. (1) and in line with the oligarchic growth picture of Kokubo \& Ida (2000), which we assume to be a reasonable description of the state of the inner disk at 0.5 Myr. Data for this initial disk model are shown in Table 1 which gives, for zones interior and exterior to the snowline, values for the total mass of solid material $M_{\text {solid }}$, the number and mass of protoplanets $N$ and $m_{\text {proto }}$, and the number and mass of super-planetesimals $N^{\prime}$ and $m_{\mathrm{s}-\mathrm{pl}}$. Note that as superplanetesimals act as statistical tracers for a much more numerous population of real planetesimals, their mass is much greater than that of a real planetesimal, but for the purposes of calculating gas drag, each super-planetesimal is treated as if it is a single $10 \mathrm{~km}$ radius object of realistic mass. The parameter $f_{\text {proto }}$, at the foot of Table 1, is the mass fraction of the solids disk contained in protoplanets and we use this here as a rough measure of the evolution of the disk, taking $f_{\text {proto }}=0.5$ to denote the transition between oligarchic and giant impact growth regimes.

From $t=0$, we run our new model for $0.1,0.25,0.5,1.0$ and $1.5 \mathrm{Myr}$ in the absence of the giant, with $\tau_{\text {nbody }}=8$ days and a simulation inner edge of $r_{*}=0.1 \mathrm{AU}$. (Note that $r_{*}$ does not necessarily denote the physical radius of the central star, but it is the distance interior to which particles are removed from the simulation and their masses added to the central star.) The evolution of the nebular mass over this time span and the particular nebular mass at each of these five epochs are shown in Fig. 1. The gas surface density profiles resulting at these times are shown in Fig. 2. It is apparent that gas drains onto the central star very rapidly at first, as the density gradient relaxes to a 


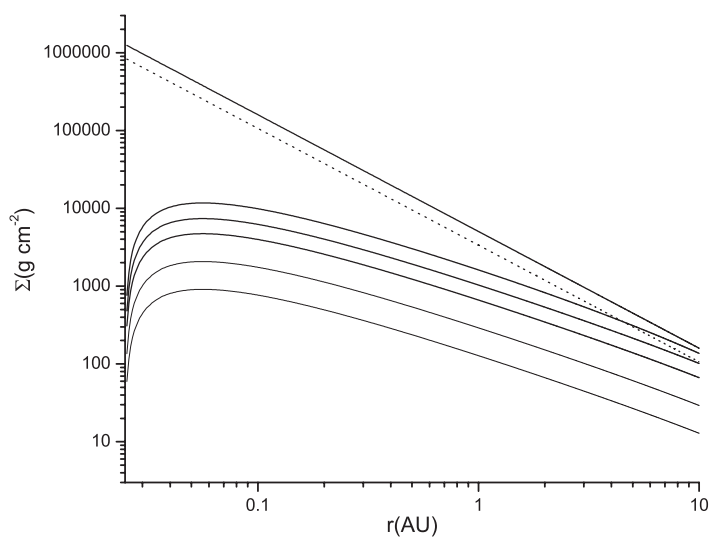

Fig. 2. Evolution of the gas surface density within the inner $10 \mathrm{AU}$ of our simulated disk. The upper solid line is the $r^{-1.5} \Sigma_{\mathrm{g}}$-profile for a $3 \times$ MMSN disk. The lower solid curves, in descending order, are the profiles at $0.1,0.25,0.5,1.0$ and $1.5 \mathrm{Myr}$ respectively. The dashed line is the fixed $\Sigma_{\mathrm{g}}$-profile assumed in Paper I.

Table 2. Overall solids disk data: after 0.1-1.5 Myr of evolution.

\begin{tabular}{c|ccccc}
\hline \hline Time (Myr) & 0.1 & 0.25 & 0.5 & 1.0 & 1.5 \\
Scenario ID & I & II & III & IV & V \\
\hline$M_{\text {solid }}\left(M_{\oplus}\right)$ & 24.8 & 24.8 & 24.8 & 24.8 & 24.8 \\
$m_{\max }\left(M_{\oplus}\right)$ & 0.29 & 0.37 & 0.63 & 1.40 & 2.13 \\
$N$ & 50 & 47 & 44 & 38 & 34 \\
$N^{\prime}$ & 4031 & 3665 & 3036 & 2342 & 1964 \\
$f_{\text {proto }}$ & 0.19 & 0.26 & 0.37 & 0.51 & 0.59 \\
\hline
\end{tabular}

shallower profile. Compared to the unevolved profile, order of magnitude reductions in gas density occur within the disk's inner regions. Data for the evolved solid components are given in Table 2 and include the values of $m_{\max }$, the mass of the largest protoplanet to have evolved in each case. (In contrast to Paper I we do not run our model to $3.0 \mathrm{Myr}$, as there remains too little gas at this time to provide for our giant planet's envelope.) The advance of planetary growth with time is indicated by the progressive increase of $m_{\max }$ and $f_{\text {proto }}$ and the reduction in particle numbers as super-planetesimals are accreted and protoplanets merge. When compared to the equivalent data from Paper I, we note that no planetesimals are lost beyond the inner edge of the simulation because of much lower gas densities near the central star.

The five type II migration scenarios studied here are constructed from the five evolved disks indicated in Fig. 1 and summarized in Table 2. Scenarios I-III take place whilst the solids disk remains in its oligarchic growth phase $\left(f_{\text {proto }}<0.5\right)$ whereas Scenarios IV-V have just entered the final giant impact stage of growth $\left(f_{\text {proto }}>0.5\right)$. A giant planet of mass $0.5 M_{\mathrm{J}}$ is placed into each simulation at 5.0 AU after removing $0.4 M_{\mathrm{J}}$ of gas from between 3-7 AU. The giant then proceeds to clear out an annular gap in the disk and migrates inward according to the method described in Sect. 2.1. The runs are halted once the giant reaches $0.1 \mathrm{AU}$ (see Fig. 3). For Scenarios I-III, this takes 110000-120000 years, close to the prediction of Eq. (6). The process takes longer to complete in the cases of Scenarios IV-V ( $\sim 150000$ and 220000 years respectively) because by the time of the appearance of the giant planet the gas disk is substantially depleted and is less effective at driving migration. In order to better model processes when the giant migrates down to small radial distances, we contract the simulation inner edge down to a realistic $\mathrm{T}$ Tauri star radius: $r_{*}=0.014 \mathrm{AU} \cong 3 R_{\odot}$.

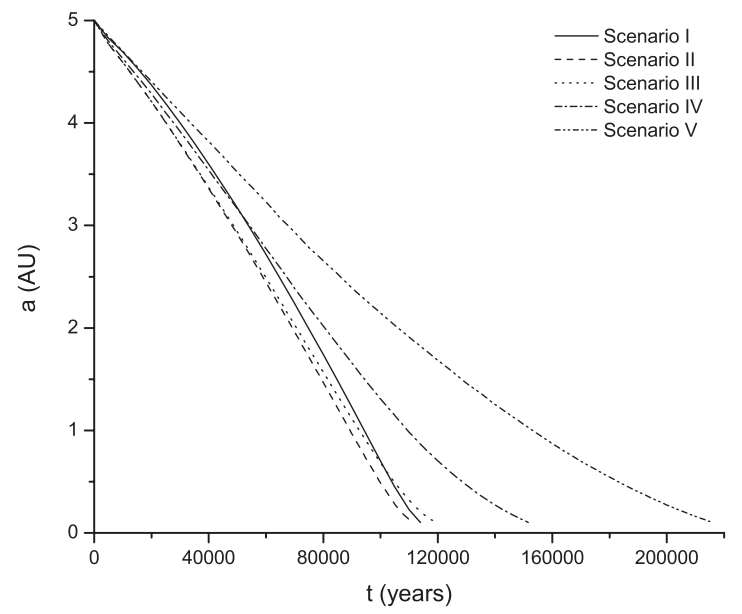

Fig. 3. Semi-major axis evolution of the giant planet in each scenario from the launch time (the top row in Table 2) to the time at which it arrives at $0.1 \mathrm{AU}$.

The initial timestep chosen for the symplectic integrator was $\tau_{\text {nbody }}=8$ days, but it was necessary to reduce this at late times as material is driven into closer orbits. Hence each scenario was divided into a number of sequential sub-runs with $\tau_{\text {nbody }}$ being adjusted at each re-start so as to keep the timestep close to one tenth the orbital period of the innermost object. Since planetesimals in this new model suffer less orbital decay due to gas drag, it was possible to conduct these runs with a higher value of $\tau_{\text {nbody }}$ than in Paper I. However, this advantage was negated at late times as the adaptive $\tau_{\text {gas }}$ falls steeply as the giant planet moves within $1 \mathrm{AU}$. From $t=0$, the scenarios presented here each required 4-8 weeks of $2.8 \mathrm{GHz}$ CPU-time for completion.

\section{Results}

We begin describing the results by focussing on the evolution during the migration of the giant planet, discussing one specific case in detail before examining differences between the various runs. We then go onto describe the evolution of Scenario $\mathrm{V}$ after giant planet migration has halted, focussing on the issue of terrestrial planet formation in the scattered disk, and the likely composition of planets that form there.

\subsection{Evolution during giant planet migration}

The results of all scenarios showed a number of behavioral features in common. As in Paper I, we illustrate these first by describing the results of Scenario I in detail. We then proceed to examine how the results differ between scenarios (dependence on disk maturity) and how the results differ from those of Paper I (dependence on an evolving gas disk).

\subsubsection{Typical features of a run}

The typical effects of a migrating giant planet on an inner solids disk observed from Paper I were as follows: 1) shepherding of planetesimals; 2) capture of objects at first order mean motion resonances; 3) acceleration of accretion interior to the giant with possible hot-Neptune formation; and 4) generation of a scattered exterior disk. To a greater or lesser extent, these features were also observed in our new simulations. Four snapshots of the evolution of Scenario I are illustrated in Figs. 4-7 showing the mass, inclination and eccentricity of objects, and the gas surface 

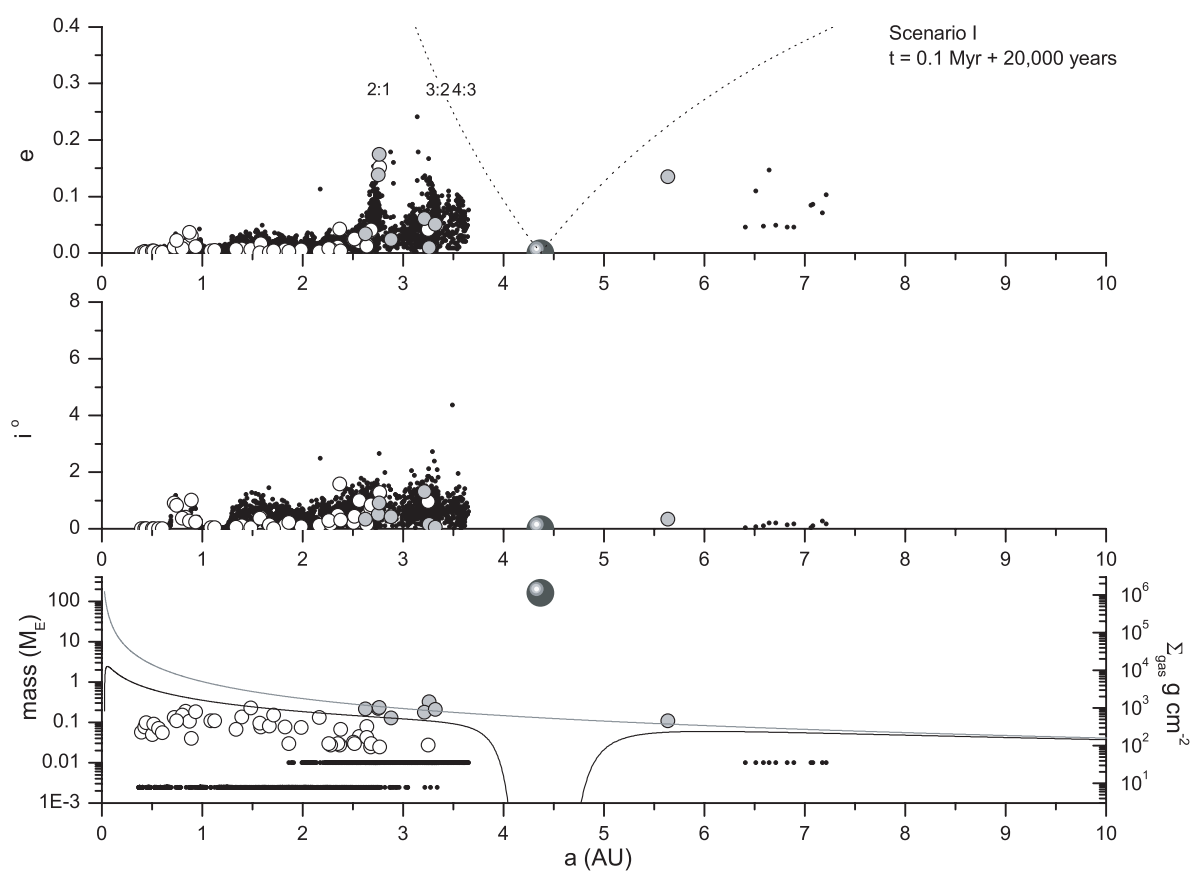

Fig. 4. Scenario I at 20000 years after the start of giant planet migration, showing the mass, inclination and eccentricity of objects. Small black dots represent super-planetesimals; white filled circles are rocky protoplanets; grey filled circles are icy protoplanets and the large highlighted grey filled circle is the giant. Objects plotted between the dotted lines in the upper panel have orbits that intersect the orbit of the giant. The location of the 2:1, 3:2 and 4:3 resonances with the giant are indicated. Gas surface density is read on the right hand axis of the lower panel, the upper grey curve being the unevolved profile at $t=0$ and the lower black curve being the current profile.

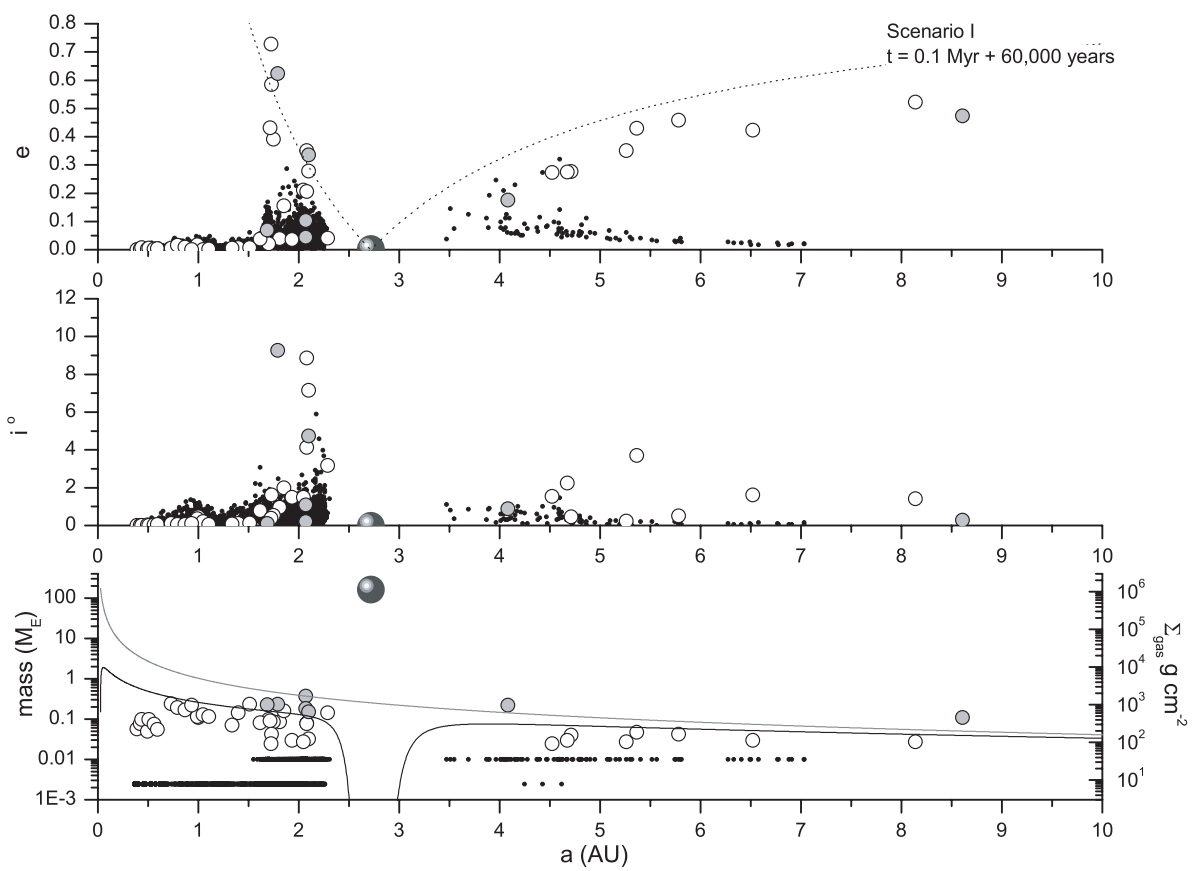

Fig. 5. Scenario I at 60000 years after the start of giant planet migration. The giant has now moved inward to 2.72 AU. Increasing excitation of the orbits of protoplanets captured at resonances is apparent, as is the build-up of matter scattered into external orbits.

density vs. semi-major axis. The original provenance of the protoplanets (interior or exterior to the snowline) is denoted by the shading of its symbol as described in the caption to Fig. 4. In the case of a merger between rocky and icy protoplanets, this shading is determined by that of the most massive of the pair.

An early stage in the evolution of Scenario I, 20000 years after the introduction of the giant planet, is shown in Fig. 4. The giant has opened a $\sim 0.75 \mathrm{AU}$ gap in the gas and has migrated inward to $4.37 \mathrm{AU}$, shepherding the outer disk edge at the $4: 3$ resonance, now at 3.61 AU. Capture of objects at the 3:2 and 2:1 resonances, at 3.33 and $2.75 \mathrm{AU}$ respectively, is apparent from eccentricity spikes visible in the upper panel and a clustering of protoplanets in the lower panel. Even at this early phase, before the giant has entered the original confines of the interior disk, one protoplanet and a handful of super-planetesimals have been scattered into external orbits.
The system midway through the run, 60000 years after the introduction of the giant planet, is shown in Fig. 5. The giant has now migrated to $2.72 \mathrm{AU}$, putting the positions of the $3: 2$ and $2: 1$ resonances at 2.07 and $1.71 \mathrm{AU}$ respectively. Strong excitation of protoplanetary orbits is now apparent at these locations, as is the build-up of scattered material in external orbits. The primary mechanism of this expulsion is evident from the behavior of material captured at resonances. Continuous resonant pumping results in orbits becoming eccentric enough to eventually intersect the orbit of the giant planet. A series of close encounters with the giant then follows, eventually resulting in a final encounter where the object is accreted or expelled into a non-intersecting exterior orbit.

An advanced stage of Scenario I, 100000 years after the introduction of the giant planet is shown in Fig. 6. The giant planet is now at $0.70 \mathrm{AU}$ and the $3: 2$ and 2:1 resonances are at 0.54 and 

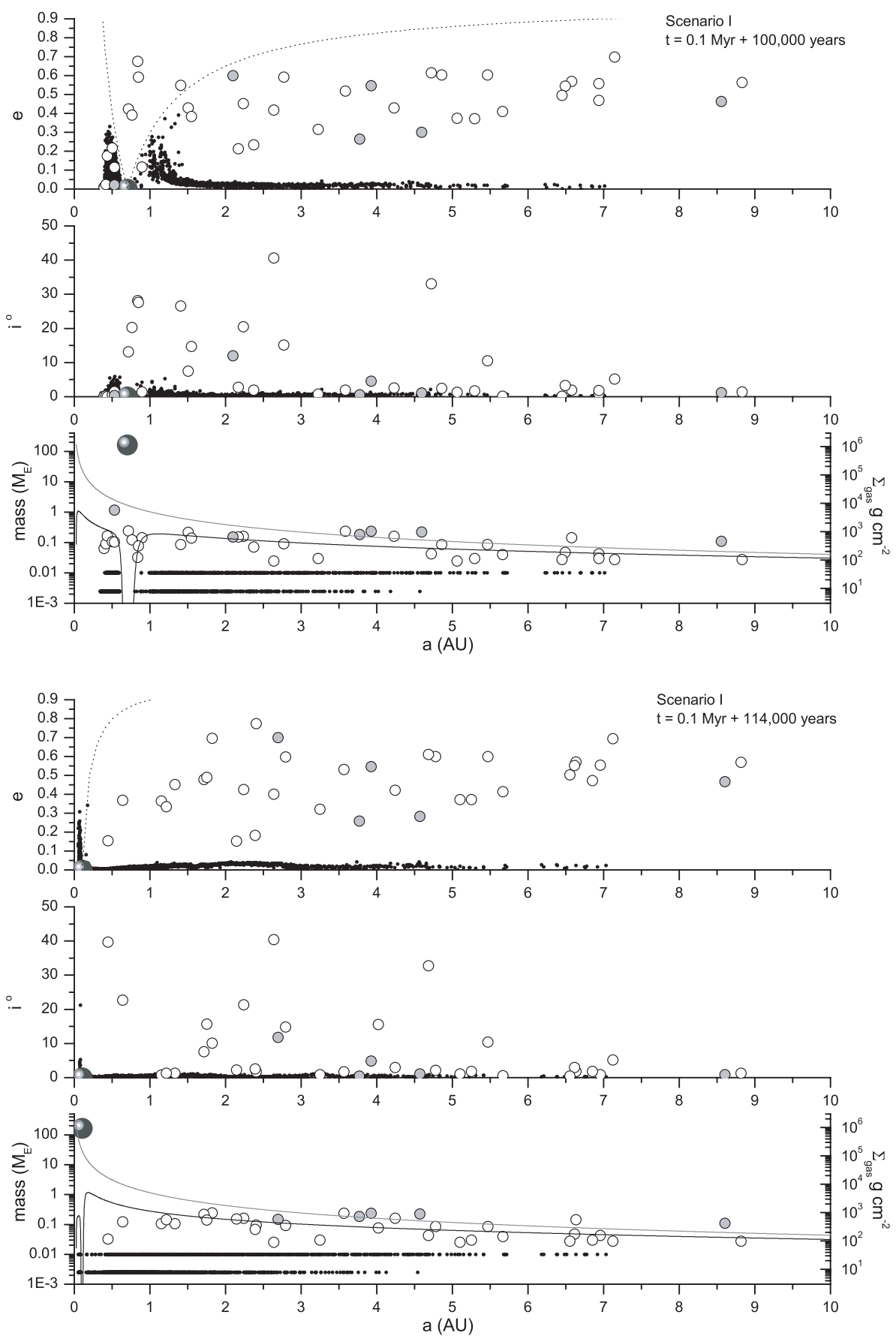

Fig. 6. Scenario I at 100000 years after the start of giant planet migration. The giant has now moved inward to $0.70 \mathrm{AU}$. Five protoplanets are currently crossing the orbit of the giant. The scattered disk has grown and a $>1 m_{\oplus}$ planet is accreting within the compacted interior disk.
Fig. 7. Scenario I at 114000 years after the start of giant planet migration. The giant planet has migrated to $0.1 \mathrm{AU}$. Most interior mass has been lost after the most massive interior protoplanet impacts the giant. $63 \%$ of the original solids disk mass now resides in exterior orbits.
0.44 AU respectively. A substantial scattered external disk has now formed and sufficient gas remains in this early scenario to rapidly damp the orbits of scattered planetesimals. An impression of the scattering process in action is given by the five protoplanets currently crossing the giant's orbit. The interior disk is compacted to high surface densities, but now that strong first order resonances with the giant are influential throughout its width, and gas densities have fallen by a factor of $>10$, there is a noticeable dynamical stirring of its entire remaining population. Nevertheless, accretion has speeded up in this shepherded zone with the growth of one protoplanet of $1.17 m_{\oplus}$ at $0.53 \mathrm{AU}$ outrunning that of its neighbors.

Scenario I is terminated at 114000 years after the start of migration when the giant planet arrives at $0.1 \mathrm{AU}$ and the system is illustrated at this point in Fig. 7. Two thirds of the original solids disk mass has survived the migration episode - the great majority of this residing in the scattered exterior disk. Most of the remainder has been accreted by the giant planet. Just $\sim 4 \%$ of the original disk mass remains interior to the giant and none of this is in the form of large bodies. The rapidly accreting interior protoplanet seen in Fig. 6 continued its inward progress close to the $3: 2$ resonance position with its orbit being welldamped by strong collisional damping and dynamical friction from planetesimals and smaller protoplanets (see Fig. 10 in Sect. 3.1.4). However, 2000 years before its demise, the protoplanet drifted outward and became captured at the 4:3 resonance. At this location, both accretion and dynamical friction were reduced allowing the protoplanet's orbit to become progressively more eccentric. At 109220 years the protoplanet, now weighing in at $2.41 m_{\oplus}$, collided with and was accreted by the giant. The 


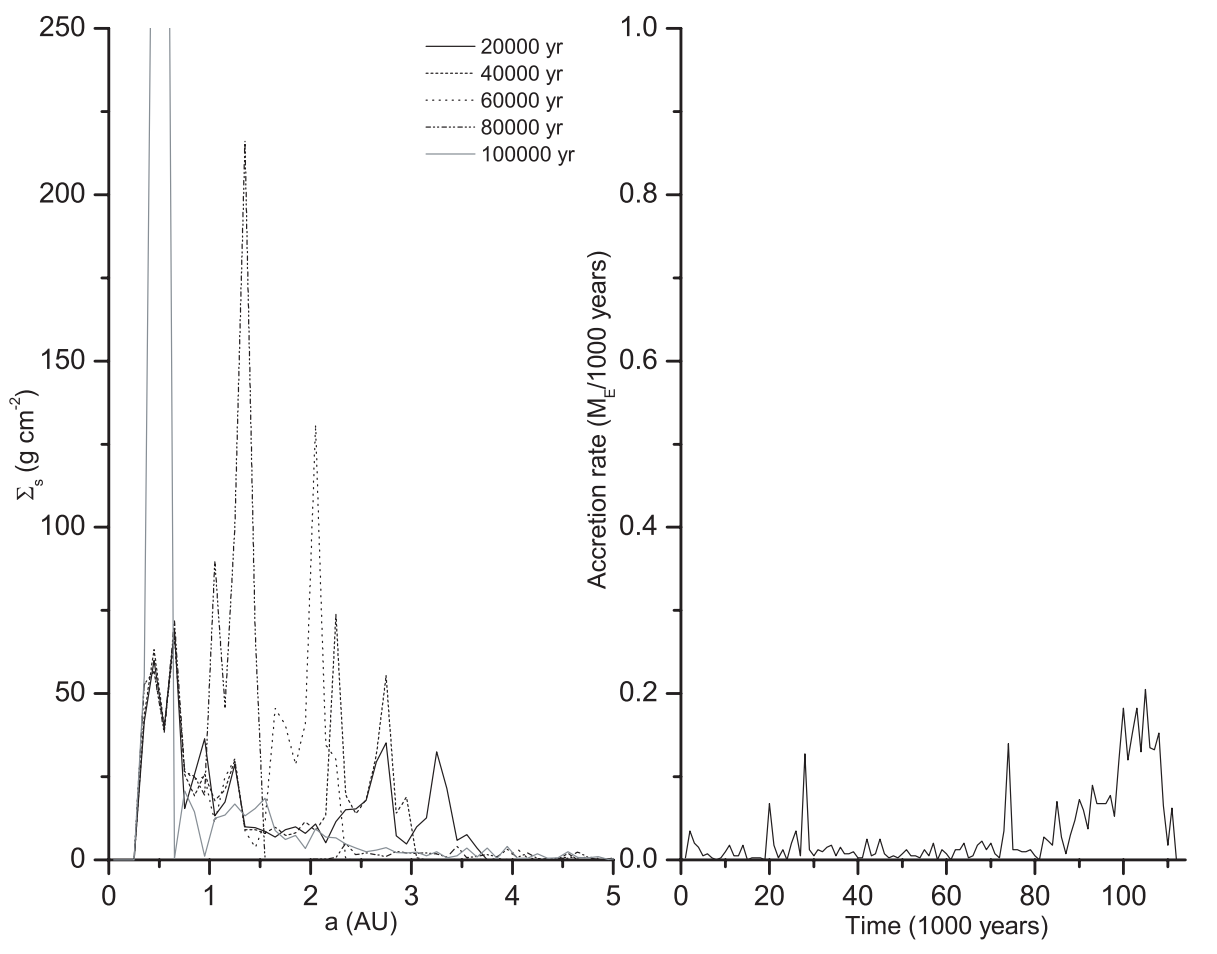

Fig. 8. Surface density evolution (left hand panel) and accretion rates (right hand panel) for Scenario I. Growing surface density peaks at the $2: 1$ and $3: 2$ resonances sweep through the inner system ahead of the giant. Accretion rates increase after $\sim 80000$ years within the compacted portion of the disk. five other less massive interior planets visible in Fig. 6 grew very little, remaining between $\sim 0.03-0.2 m_{\oplus}$, and in due course one of them impacted the giant and four were scattered.

To emphasize the above description of Scenario I, the surface density evolution of the disk and its accretion rate are shown in Fig. 8. The left hand panel shows the disk surface density profile (obtained by summing all protoplanets and super-planetesimals in $0.1 \mathrm{AU}$ width bins) at 20000, 40000, 60000, 80000 and 100000 years after the start of migration; the right hand panel plots the amount of mass accreted onto protoplanets only (including protoplanet mergers) every 1000 years for the duration of the run. In the $\Sigma_{\text {s }}$ plot, two surface density enhancements are clearly visible as spikes at the 3:2 and 2:1 resonances and are seen to grow whilst moving inward. At 80000 years, these have almost merged into one: the shepherded portion of the original disk having by now been squeezed into a dense ring. By 100000 years, most of this mass is now confined within $0.6 \mathrm{AU}$ and $\Sigma_{\mathrm{s}}$ here has risen to $\sim 500 \mathrm{~g} \mathrm{~cm}^{-2}$ which is off the vertical scale in the figure. This amounts to an increase by a factor of $\sim 10$ over the previous, undisturbed, disk surface density, but is only about half the increase seen in the equivalent Scenario presented in Paper I. The effect of this disk compaction process is visible in the accretion rate plot. Mass accretion rises significantly after 80000 years due to both the high values of $\Sigma_{\mathrm{s}}$ and the fact that much of this mass now resides in a zone where dynamical times are shorter. However, the large, terminal, accretion rate spike described in Paper I is not reproduced here (compare Fig. 8 with Fig. 6 of Paper I). This is because close to the end of that previous simulation a $15.65 m_{\oplus}$ hot-Neptune was assembled in a dramatic phase of runaway accretion interior to $0.1 \mathrm{AU}$. In the case presented here the compacted interior disk is only half as dense and is much less well damped (note the large difference between the upper and lower gas density curves in Fig. 7) and, whilst a protoplanet does grow to $2.41 m_{\oplus}$ in this region, as described above, it does not survive and is accreted by the giant planet. We describe the formation and fate of interior planets in more detail in Sect. 3.1.4.

\subsubsection{Dependence on the maturity of the inner disk}

The reason for running five scenarios through a progressively more mature inner disk is to see if the timing of migration has any systematic effect on the results. This is possible as the partitioning of the solids disk between inner and outer remnants is influenced by the level of damping that particles are subject to, which declines with age. In Paper I, where $\Sigma_{\mathrm{g}}(r)$ is fixed, this occurs as a side effect of accretion: as planetesimals are accreted by protoplanets ( $f_{\text {proto }}$ increases), fewer small particles remain that are subject to gas drag and which can exert dynamical friction. In these latest simulations, since we now have an evolving gas disk, the strength of gas drag on susceptible particles also declines with time and is particularly marked close to the central star and giant planet.

Data describing the fate of the solids disk mass at the end of each scenario are shown in Table 3. Disk mass that is lost is either accreted by the central star, ejected from the system, or accreted by the giant planet; that which survives is partitioned between bodies orbiting interior or exterior to the final orbit of the giant planet at $0.1 \mathrm{AU}$.

In all scenarios, a negligible quantity of mass was ejected or lost to the central star. However, a significant fraction of the disk mass (14-33\%) was accreted by the giant, especially towards the end of the migration. At these late times, planetesimals are shepherded into the partially evacuated inner regions of the gas disk where gas drag is less effective at damping orbital perturbations from the giant and growing protoplanets. Once a planetesimal strays into the annular gap in the gas containing the giant, gas drag vanishes and accretion or scattering by the giant follows. This increased excitation of the shepherded planetesimal population, and the thinning down of their number, renders them less effective at damping protoplanetary orbits via dynamical friction and collisions. Hence, at late times the orbits of the remaining interior protoplanets also tend to destabilize, with one of the same two fates in store. A trend can be seen in Table 3 for the giant planet to accrete less material with disk maturity. This occurs because as the disk ages the gas density and the solids mass 
Table 3. Fate of the disk mass at the end of each run.

\begin{tabular}{cccccc}
\hline \hline Scenario & I & II & III & IV & V \\
\hline Total Initial Solids $\left(M_{\oplus}\right)$ & 24.81 & 24.81 & 24.81 & 24.81 & 24.81 \\
Total Surviving Solids $\left(M_{\oplus}\right)$ & $16.60(67 \%)$ & $16.69(67 \%)$ & $17.40(70 \%)$ & $21.23(86 \%)$ & $20.22(81 \%)$ \\
Interior Surviving Solids $\left(M_{\oplus}\right)$ & $0.88(4 \%)$ & $0.65(2 \%)$ & $1.00(4 \%)$ & $0.84(3 \%)$ & $0.31(1 \%)$ \\
$N, f_{\text {proto }}$ & 0,0 & 0,0 & 0,0 & 0,0 & 0,0 \\
Exterior Surviving Solids $\left(M_{\oplus}\right)$ & $15.72(63 \%)$ & $16.04(65 \%)$ & $16.40(66 \%)$ & $20.39(82 \%)$ & $19.90(80 \%)$ \\
$N, f_{\text {proto }}$ & $39,0.27$ & $29,0.28$ & $33,0.42$ & $31,0.63$ & $23,0.66$ \\
Accreted by Star $\left(M_{\oplus}\right)$ & $0.01(0.04 \%)$ & $0.01(0.04 \%)$ & $0.11(0.4 \%)$ & $0.0(0 \%)$ & $0.0(0 \%)$ \\
Accreted by Giant $\left(M_{\oplus}\right)$ & $8.20(33 \%)$ & $7.85(32 \%)$ & $6.77(27 \%)$ & $3.41(14 \%)$ & $4.59(19 \%)$ \\
Ejected $\left(M_{\oplus}\right)$ & $0.00(0 \%)$ & $0.26(1 \%)$ & $0.51(2 \%)$ & $0.17(1 \%)$ & $0.0(0 \%)$ \\
\hline
\end{tabular}

fraction in small bodies both decline, resulting in less dynamical dissipation of both planetesimals and protoplanets. Less matter is shepherded in such mature disks so there is less of an interior remnant for the giant to accrete from at late times.

In all scenarios, a large majority of the disk solids are found to survive the migration episode - over two thirds of the original inventory. Table 3 shows that there is essentially no trend with disk maturity in the partitioning of surviving mass between interior and exterior remnants. Just a few percent of the mass remains interior to the giant in all cases. When the giant planet migrates through a disk undergoing oligarchic growth $\left(f_{\text {proto }}<0.5\right.$; Scenarios I-III) $\sim 65 \%$ of the original disk mass survives by being scattered into the exterior disk. This fraction increases for disks undergoing giant impact-style growth $\left(f_{\text {proto }}>0.5\right.$; Scenarios IV-V) to $>80 \%$, not because more mass remains at $<0.1 \mathrm{AU}$ but because less mass is accreted by the giant at late times.

\subsubsection{Dependence on an evolving gas disk}

The salient dynamical behaviors of solids disk particles such as shepherding, resonant capture, scattering by the giant planet and eventual partition into interior and exterior remnants are observed generally in the results of both the present model and those of Paper I. However, the introduction of an evolving gas disk causes the relative predominance of these outcomes to differ. This is because both the principal sources of dissipation, dynamical friction and gas drag, fall with time, whereas in the model from Paper I only the former declines. There are five systematic differences between the results of Paper I and those presented here.

1 Much less mass is lost to the central star.

2 Much more mass is accreted by the giant planet.

3 Disk partitioning between inner and outer remnants is much less sensitive to disk maturity.

4 The protoplanet mass fraction $\left(f_{\text {proto }}\right)$ in the exterior scattered disk is lower.

5 Interior hot-Neptune-type planets grow to smaller masses and do not survive.

The reasons for the first two items are the large reductions in gas density close to the central star and in the vicinity of the giant planet. Planetesimals do not have time to spiral into the central star and feel little eccentricity damping when close to the giant because of the low gas drag in these regions. The reasons for the last three items stem from the fact that the system is less dissipative so scattering behavior predominates over shepherding at all epochs. Interior remnants are consistently much smaller than outer remnants which always include $>60 \%$ of the original solids disk mass. The increased tendency for planetesimals to scatter means that there is less of a fractionation of planetesimals from

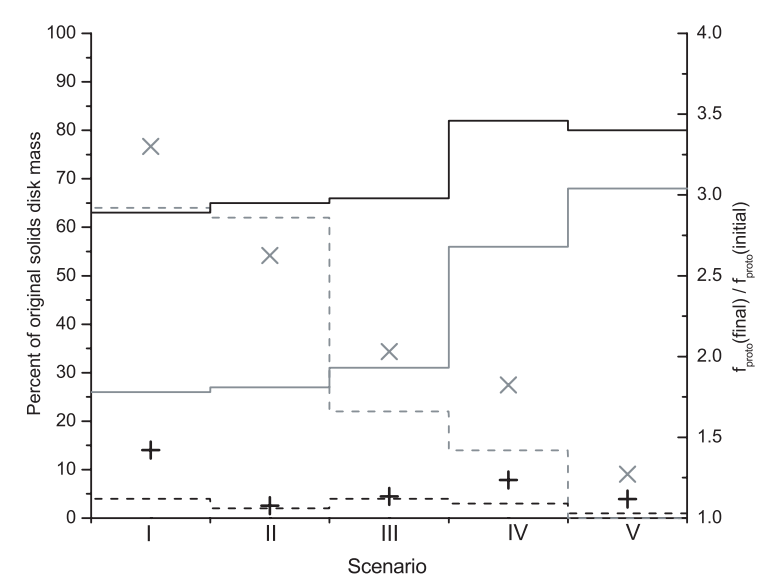

Fig. 9. Comparison of the results of Paper I (grey lines and $\times$ symbols) and the results of this paper (black lines and + symbols). Dashed lines and solid lines are the percentage of original disk mass found in the interior and exterior remnants respectively. The ratios of the protoplanet mass fraction in the final exterior disk to that of the original disk, $f_{\text {proto }}$ (final) $/ f_{\text {proto }}$ (initial), are indicated by the $\times$ and + symbols and are read off the right hand $y$-axis.

protoplanets between interior and exterior remnants. More planetesimals are expelled into the exterior disk and hence its overall mass fraction contained in protoplanets $\left(f_{\text {proto }}\right)$ is lower.

These tendencies are illustrated in Fig. 9 where the percent of the original solids disk mass surviving at the end of the simulations presented here are compared with the simulation results from Paper I. Interior and exterior remnants are plotted as dashed and solid lines respectively; gray lines are the results of Paper I and black lines are those of the present work. It can be seen that partitioning varies strongly with disk maturity in the case of a steady-state gas model and weakly, if at all, when the nebula gas is allowed to viscously evolve. Since gas drag does not decline in the former case, the shepherding of planetesimals is more influential and more mass remains in the interior remnant in early scenarios, which is mostly contained in surviving hot-Neptunetype planets. Late scenarios from Paper I behave more similarly to the ones presented here as a greater fraction of the solids disk mass is contained within protoplanets, which do not feel the gas, and their source of dissipation, dynamical friction, is weaker due to a decline in super-planetesimal numbers ${ }^{1}$.

The increased tendency in the present model for planetesimals to scatter into the exterior disk can also be appreciated from Fig. 9 by comparing, for the two models, the ratio of the protoplanet mass fraction in the final exterior disk to that of the original disk, $f_{\text {proto }}($ final $) / f_{\text {proto }}$ (initial). If the scattering process

1 Note that Scenario V is less comparable to its counterpart in Paper I as it is initiated at a different time, 1.5 Myr earlier. 


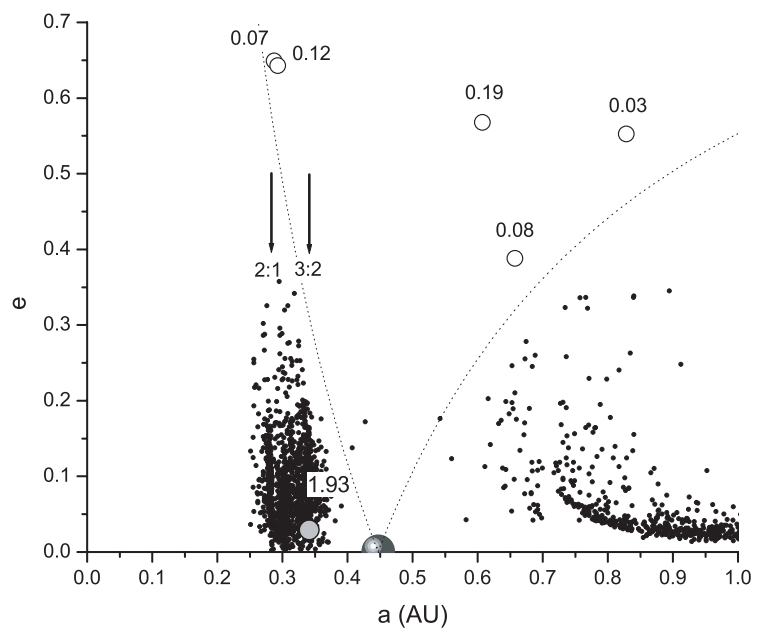

Fig. 10. Blow-up of the inner $1 \mathrm{AU}$ of Scenario I, showing eccentricity vs semi-major axis 105000 years after the start of giant planet migration. Protoplanetary masses are indicated in $m_{\oplus}$ and the locations of the 2:1 and 3:2 resonances are shown with arrows. The giant planet is at $0.45 \mathrm{AU}$ and our hot-Earth candidate is a $1.93 m_{\oplus}$ planet at $0.34 \mathrm{AU}$ with $e \approx 0.03$. Five lower mass protoplanets are in the process of being scattered into the exterior disk. (Compare with Fig. 6 which shows the situation 5000 years earlier.) As described in Sect. 3.1.1, the hot-Earth candidate is eventually accreted by the giant planet $\sim 4000$ years later once it has migrated further inward to $\sim 0.26 \mathrm{AU}$.

does not discriminate between protoplanets and planetesimals, and there is no further accretion after scattering, this ratio would remain at $f_{\text {proto }}$ (final $) / f_{\text {proto }}($ initial $)=1$. Subsequent accretion will raise this ratio, but not by much over the timescale considered, especially as accretion rates are reduced in the exterior disk due to its dynamically excited state. Reading from the right hand y-axis, Fig. 9 shows that $f_{\text {proto }}($ final $) / f_{\text {proto }}$ (initial) $<1.5$ for all of the present model results whereas much higher values are obtained from Paper I. Protoplanets are preferentially scattered into the exterior disk when there is strong and unvarying gas drag, whereas fractionation is much less marked when gas drag declines over time and falls to near zero in the vicinity of the giant planet.

\subsubsection{Formation and survival of hot-Neptunes}

A striking feature of the results of Paper I was the growth and survival, interior to the final orbit of the giant, of hot-Neptune and hot-Earth type planets ranging between $\sim 2-16 m_{\oplus}$. No such planets are found to survive in the runs of our present model. Accelerated accretion in the shepherded disk is observed with some protoplanets growing to $\sim 1-3 m_{\oplus}$ (see Figs. 6 and 8), but in each scenario their eventual fate was to impact the giant planet just before the end of the simulation. After formation, these planets become locked in a mean motion resonance (3:2 or $4: 3$ ) with the giant, and subsequent eccentricity pumping during migration leads to collision and merger with the giant shortly before migration halts. However, had we halted giant planet migration earlier, at $\sim 0.25-0.5 \mathrm{AU}$ instead of $0.1 \mathrm{AU}$, these simulations would predict the existence of hot-Earths (e.g. see Fig. 10).

We have performed additional calculations, very similar to those presented here, in which we switched off the effect of dissipating spiral waves travelling into the disk (i.e. $T(r)=0$ in Eq. (7)). This term helps create an inner cavity in the gas disk as the planet migrates inward. These calculations produced interior planets ranging in mass $\sim 2-4 m_{\oplus}$ during giant planet migration. A variety of final outcomes were noted: 1 ) some of them were accreted by the giant planet; 2 ) others were scattered externally into stable orbits at $a \sim 0.4 \mathrm{AU}$; and 3 ) a few remained in interior orbits, typically close to $0.076 \mathrm{AU}$ (the $3: 2$ resonance). These results, taken alongside the five scenarios already presented in this paper and those from Paper I show a distinct trend: a strongly dissipating gas disk interior to the giant planet leads to the formation of fairly massive, hot-Neptune-like planets which survive; a gas disk of lower density leads to the formation of lower mass interior planets that often do not survive.

We note at this point that our models currently neglect some potentially important sources of dissipation due to general uncertainty about how planetary formation can proceed in their presence, such as type I migration (Ward 1997; Tanaka et al. 2002; Tanaka \& Ward 2004) and the circularization of orbits due to stellar tides. Associated with the former is strong eccentricity and inclination damping of low mass planets which may facilitate the survival of inner planets. Simulations are currently underway to examine this possibility. Tidal damping of orbits however is unlikely to have a significant effect on our results because all shepherded protoplanets are scattered or accreted by the giant at $a \gtrsim 0.1 \mathrm{AU}$. Whilst tidal circularization times at these radii are uncertain, due to the uncertainty of $Q$, they probably range from $\sim 10^{8}-10^{10}$ yr (Goldreich \& Soter 1966), orders of magnitude greater than the millenia it takes for our giant planets to traverse the final $0.5 \mathrm{AU}$ of their migration.

\subsubsection{The exterior scattered disk}

In Paper I it was noted that the exterior solids disks generated by the giant planet migration were dynamically excited, depleted of planetesimals, and spread over a greater radial extent than the original disk. Whilst subsequent planet formation still seemed possible in such a disk, it was predicted to occur over significantly extended timescales. However, in the Paper I scenarios only $\sim 1-2 m_{\oplus}$ of planetesimals were scattered into the exterior disk, whereas in the present model this quantity rises to $\sim 8-11 m_{\oplus}$. In addition, planetesimals are not scattered as widely as protoplanets and their excited orbits damp rapidly when remote from the influence of the giant planet. Whilst the decline in gas drag with time lessens this trend in later scenarios, in all cases the inner regions of the scattered disk remain well populated.

There is a greater similarity between the current models in the outcome of scattering of the protoplanetary population and data for the external protoplanets are shown in Table 4, giving their number, mean and maximum masses and orbital inclinations, and their mean, minimum and maximum semi major axes and eccentricities. As expected, protoplanetary numbers fall and masses rise with disk maturity, an effect largely due to prior accretion before the appearance of the giant planet. Allowing for stochastic events, such as giant impacts and strong scatterings, these data are much the same in Paper I, as are the mean semimajor axes and eccentricities $(\bar{a} \approx 5 \mathrm{AU}, \bar{e} \approx 0.5)$. However the minimum values of the semi-major axes and eccentricities $\left(a_{\min }\right.$ and $\left.e_{\min }\right)$ are lower than in Paper I. This is because lateshepherded protoplanets tend to scatter rather than assembling into hot-Neptunes and their resultant orbits damp more rapidly as many more planetesimals are available to exert dynamical friction. 
Table 4. Data describing the external surviving protoplanets at the end of giant planet migration.

\begin{tabular}{cccccccccccc}
\hline \hline Scenario & $N$ & $\bar{m}_{\text {proto }}\left(M_{\oplus}\right)$ & $m_{\max }\left(M_{\oplus}\right)$ & $\bar{a}(\mathrm{AU})$ & $a_{\min }(\mathrm{AU})$ & $a_{\max }(\mathrm{AU})$ & $\bar{e}$ & $e_{\min }$ & $e_{\max }$ & $\bar{i}^{\circ}$ & $i_{\max }^{\circ}$ \\
\hline I & 39 & 0.11 & 0.25 & 5.43 & 0.45 & 37.92 & 0.51 & 0.15 & 0.99 & 8.85 & 40.45 \\
II & 29 & 0.16 & 0.74 & 4.99 & 0.71 & 15.25 & 0.49 & 0.045 & 0.91 & 8.59 & 35.45 \\
III & 33 & 0.21 & 0.64 & 4.68 & 0.37 & 12.22 & 0.46 & 0.059 & 0.89 & 8.32 & 43.97 \\
IV & 31 & 0.41 & 2.35 & 5.16 & 0.43 & 8.92 & 0.50 & 0.086 & 0.94 & 5.81 & 41.93 \\
V & 23 & 0.57 & 2.19 & 6.88 & 0.49 & 18.18 & 0.45 & 0.079 & 0.83 & 3.53 & 16.24 \\
\hline
\end{tabular}

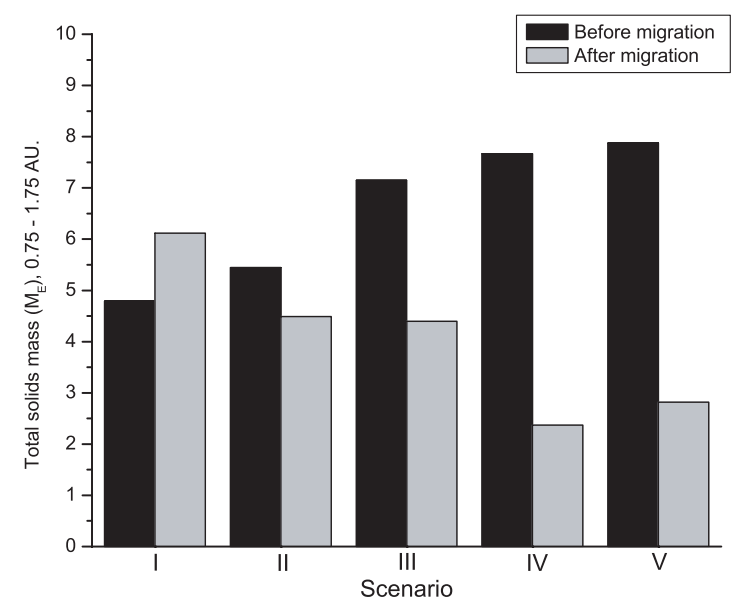

Fig. 11. The total solids mass between $0.75-1.75 \mathrm{AU}$, before and after the giant planet migration, plotted for each scenario.

\subsection{Post-migration terrestrial planet formation}

What do the results of the five scenarios presented here have to say about the probablity of forming terrestrial planets in the scattered disks? Considering the Scenarios I-V overall, if the factor of long-term importance is the mass distribution, rather than initial dynamics, then such planets should form and await discovery in hot-Jupiter systems. This point is made in Fig. 11 where the total solids mass with semi-major axes between 0.75-1.75 AU, before and after giant planet migration, is plotted for each scenario. A clear trend is visible for less matter to be found in this region with increasing disk maturity as it is more widely scattered. Mass dispersal in late scenarios, however, is partially offset by the pre-existing inward evolution of material in more mature disks which enhances the mass present in inner regions. It is also offset by the fact that fairly massive protoplanets are scattered into the external disk in late Scenarios (IV and V see Table 4), so that terrestrial planet formation in the scattered disk has already received a significant boost from accretion prior to and during migration. In all cases, more than $2 m_{\oplus}$ of planet forming material remains in the "maximum greenhouse" habitable zone (Kasting et al. 1993) of the system after the passage of the giant planet.

Extending our runs for the additional 50-100 Myr it would take to form a completed external planetary system is beyond our current computational capabilities. The principal difficulty is the presence of the hot-Jupiter at $0.1 \mathrm{AU}$ which limits $\tau_{\text {nbody }}$ to an excessively low value. We have, however, extended Scenario V for $2 \mathrm{Myr}$ and find that (apparently stable) terrestrial planets $d \mathrm{o}$ form in the habitable zone of this system.

As the gas density is low when migration of the giant planet halts in Scenario V, we assume rapid removal of the remnant gas via photoevaporation and evolve the system in the absence of gas. Figure 12 shows the region between $0-4$ AU for the scattered disk of Scenario $\mathrm{V}$ at the point when the giant planet reaches $0.1 \mathrm{AU}$ (top panel). Protoplanets of substantial size have been scattered into the external disk. The result after a further 2 Myr of gas-free accretion is shown in the bottom panel. Dynamical friction is sufficient to cause general damping of protoplanetary orbits at the expense of excitation of the planetesimals. A significant accretion event has been a giant impact and merger between the 1.42 and $0.73 m_{\oplus}$ protoplanets at $\sim 1.5 \mathrm{AU}$ resulting in a $2.15 m_{\oplus}$ body at $a=1.47 \mathrm{AU}$ with $e=0.13$. This planet, which lies within the "maximum greenhouse" habitable zone, no longer crosses the orbit of any of its neighbors and is probably a long-term survivor. There has been some rearrangement of the intersecting protoplanetary orbits beyond 2 AU with two protoplanets moving outward and circularizing at $a>4 \mathrm{AU}$, off the right hand side of the figure, to compensate for the $0.79 \mathrm{AU}$ inward movement of a massive $2.2 m_{\oplus}$ planet, now at $a=2.25 \mathrm{AU}$ with $e=0.14$. This planet is currently crossing the orbit of a smaller $1.07 m_{\oplus}$ body, the long-term fate of which will probably be accretion by one of its two larger neighbors or incremental scattering by them to a safe distance. The emergence of a stable terrestrial planetary system from such a configuration seems highly probable, and this result provides a clear prediction that terrestrial planets will be found in the habitable zones of hot-Jupiter systems.

What will be the physical nature of such planets? The issue of disk and protoplanet composition after migration is discussed in detail in Sect. 3.3. Here we note, however, that the 2.15 and $2.20 m_{\oplus}$ planets observed in Fig. 12 are composed of $\sim 30 \%$ and $45 \%$ of material originating from beyond the snowline, respectively. Assuming that trans-snowline planetesimals and protoplanets contain $75 \%$ water, a naive prediction is that these planets will contain between $20-30 \%$ water by mass. The current water inventory of the Earth is estimated to be about $0.05 \%$ by mass. Evidently terrestrial planets forming in the habitable zones of hot-Jupiter systems are likely to host deep global oceans - essentially being "water-worlds" (Kuchner 2003; Léger et al. 2004; Raymond et al. 2006), even if significant loss of volatiles occurs during high-impact accretion.

This prediction about water content, however, depends on the giant planet forming out beyond the inner edge of the snowline, rather than at its inner edge. The closer to the inner edge the giant forms, the smaller the amount of volatile-rich material that will be shepherded inward, and the lower the degree of volatile enrichment experienced by terrestrial planets forming after migration of the giant.

\subsection{Migration-driven compositional mixing}

A result found in all Scenarios $\mathrm{I}-\mathrm{V}$ is homogenization of the solids disk composition. This mixing occurs as the giant planet drives material inward from the outer part of the disk whilst generating the external disk via random scattering. An example is given in Fig. 13 which shows, for Scenario I, the composition of the solids disk before migration (top panel) and that of the surviving external disk after migration (bottom panel). In each case 


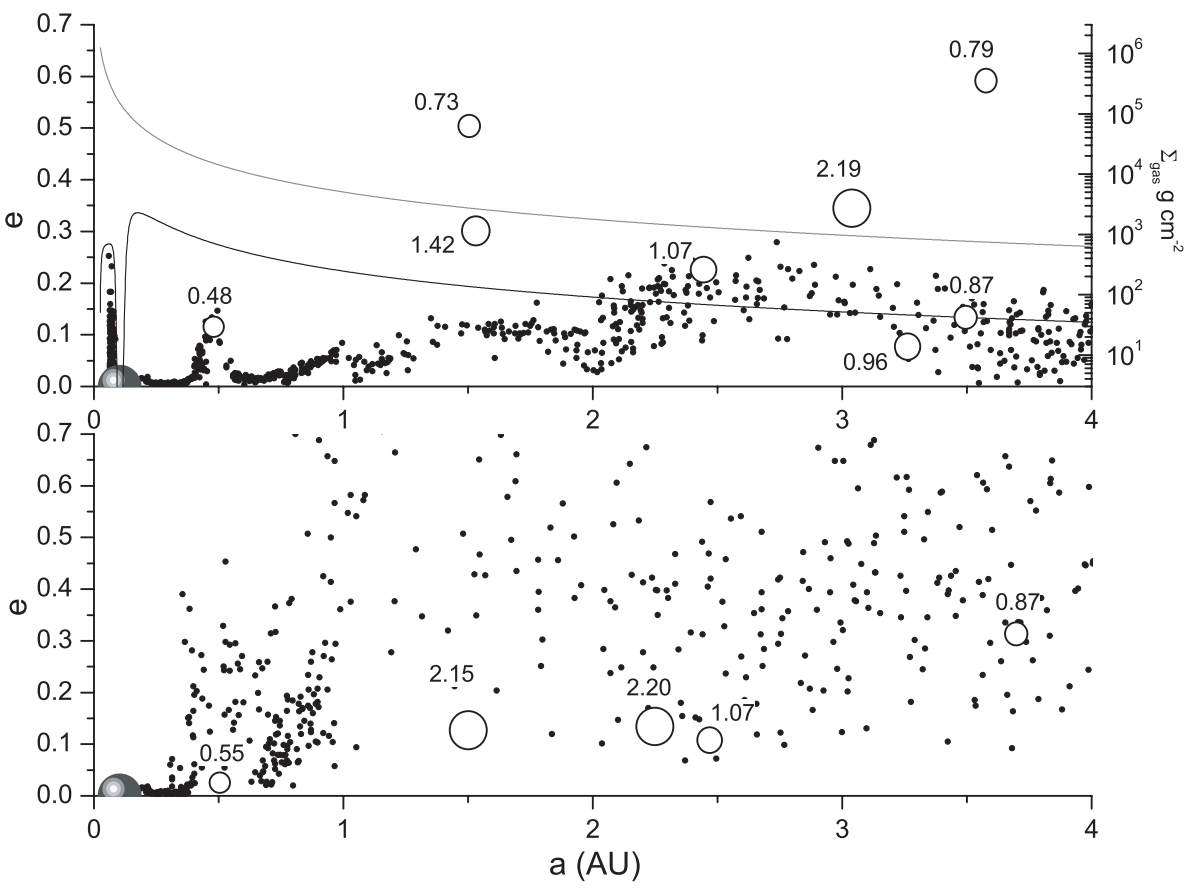

Fig. 12. Eccentricities of bodies within 4 AU at the end of Scenario V (top panel) and after a further $2 \mathrm{Myr}$ of gas-free accretion (bottom panel). Protoplanets are shown as white circles and are labeled with their mass in $m_{\oplus}$. Super-planetesimals are indicated by black dots and the grey blob at $0.1 \mathrm{AU}$ is the hot-Jupiter in its post-migration orbit. Gas density at the end of Scenario $\mathrm{V}$ is shown as the black curve in the top panel (read off the right-hand axis), the grey curve being the unevolved profile at $t=0$.

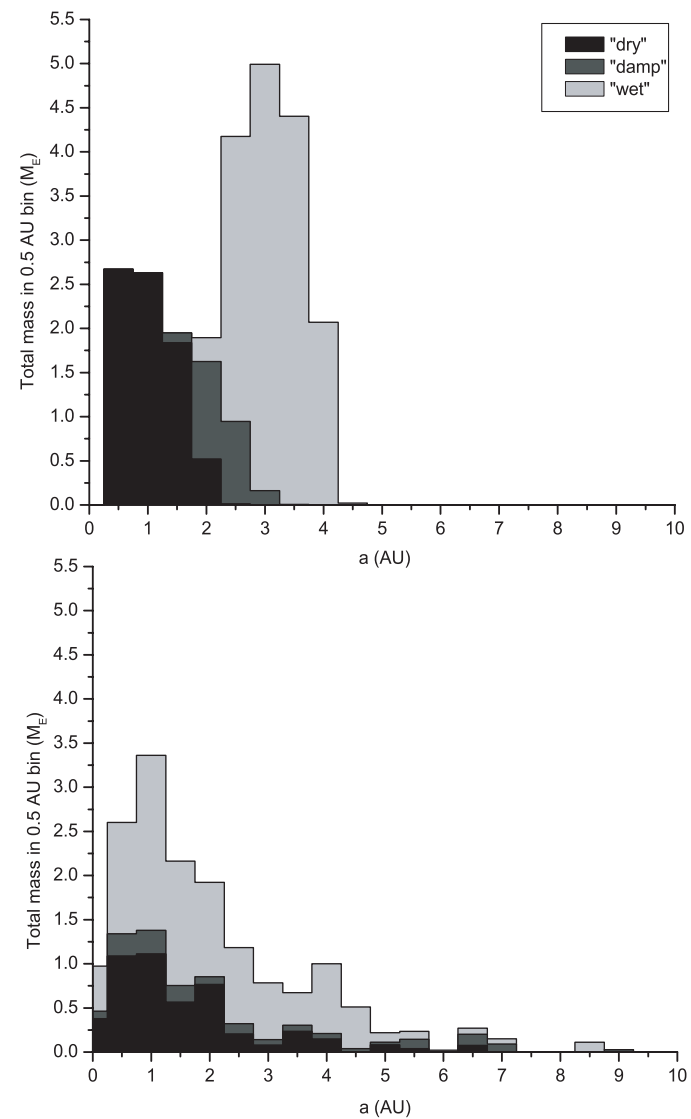

Fig. 13. Composition of the original solids disk in Scenario I (top panel) compared with the composition of the scattered disk generated through giant planet migration (bottom panel). The key is explained in the text.

the total mass of solid material is plotted in $0.5 \mathrm{AU}$ width bins with the histograms labeled "dry", "damp" and "wet" representing rocky material originating at $<2 \mathrm{AU}$, material characteristic of chondritic meteorites between 2-2.7 AU, and trans-snowline material at $>2.7 \mathrm{AU}$, respectively. It is evident that a large amount of material from beyond the snowline is shepherded into the inner regions before being left behind. Compositional mixing is similar in other scenarios, although a little less smooth in Scenarios IV \& V which have had more time to accrete substantial protoplanets from local material before the appearance of the giant planet.

In contrast to other studies of water delivery to terrestrial planets (e.g. Morbidelli et al. 2000; Chambers 2001; Raymond et al. 2004, 2005a,b, 2006), we do not assign an actual water mass fraction to our compositional phases. This is because our simulations do not extend to the completion of planetary accretion and our MMSN-type original solids surface density profile, which includes a large step-increase at $2.7 \mathrm{AU}$ (see Eq. (1)), is different from those adopted in the above-cited papers making a detailed comparison difficult. Typically, we find that at the end point for early-migration scenarios (I-III) originally dry protoplanets that have found their way into the scattered disk contain 0-25\% of trans-snowline material, but are surrounded by a large quantity of volatile-rich planetesimals from which to accrete further. In later scenarios (IV-V), where the surviving protoplanets are more mature, this range increases to 5-70\%, and whilst there is less remaining mass in small bodies to sweep up in these systems, the accretion of volatile-rich material is not yet complete.

In general we can predict that the final terrestrial planets that form in hot-Jupiter systems are likely to be much more volatilerich than the Earth, those in the habitable zone ending up with $>10 \%$ of their material originating from beyond the snowline. Accretion in the external disk, however, may pass through an early high velocity phase before completion with some protoplanets potentially losing much of their volatile inventory during giant impacts (Asphaug et al. 2006; Canup \& Pierazzo 2006). Since water-rich planetesimals are abundant throughout our external disks, however, protoplanets that have lost their original volatile endowment in a catastrophic event should be able to re-acquire some water before planetary accretion is complete. Dry terrestrial planets appear improbable in hot-Jupiter systems whereas Earth-like worlds and planets with deep global oceans (Kuchner 2003; Léger et al. 2004; Raymond et al. 2006) may well be commonplace. 


\section{Discussion}

The models presented here and in Paper I have only explored a small region of the parameter space relevant to this problem, and have inevitably adopted assumptions that simplify or omit potentially important physical processes. We discuss some of these issues below and their possible implications for our results.

(i) Giant planet mass $\mathcal{E}$ radius. In all simulations we have adopted a giant planet mass of $0.5 M_{\mathrm{J}}$, with a density of $1 \mathrm{~g} \mathrm{~cm}^{-3}$ and a radius of $0.85 \mathrm{R}_{\mathrm{J}}$. Whilst the giant planet can accrete solid matter as it migrates inward, it does not accumulate any more gas and so its mass changes by $\lesssim 5 \%$ during a run. Hot-Jupiters, however, come in a variety of masses and although their average $m \sin i$ is less than a Jupiter mass, more massive examples are known. We have not yet tested the effect of increasing the mass of our migrating giant planet, but expect that this would result in an enhanced and more widespread scattering of material into the external disk, and more accretion by the giant.

We have assumed a radius for our giant planet that is representative of a fully contracted state, which may not be realistic so soon after its formation. Giant planets of approximately Jovian mass contract to radii $\sim 2-3 R_{\mathrm{J}}$ during their rapid gas accretion phase (Papaloizou \& Nelson 2005), before cooling and contracting toward the Jovian radius over longer time scales. Adoption of a larger radius would probably result in greater accretion of solids by the giant, with gas-drag-sensitive planetesimals being preferentially accreted.

(ii) Migration halting mechanisms. The reason why hotJupiters cease their inward migration close to the central star and come to rest at $\lesssim 0.1 \mathrm{AU}$ is unknown. Various mechanisms have been suggested, such as the giant planet moving into a central magnetospheric cavity in the gas disk (Lin et al. 1996), or fortuitous dispersal of the gas disk due to photoevaporation (Trilling et al. 1998; Armitage et al. 2002; Alibert et al. 2005). We do not rely on any particular mechanism here and merely note that if the former operates then any of our scenarios may be appropriate, whereas if the latter is true then our latest scenarios (IV \& V) are more likely to be realistic as they operate at a time when most of the gas disk has already been lost. In either case, we expect an external disk to be generated during giant planet migration and renewed terrestrial planet formation to follow. The dynamical state of this scattered disk, however, will be affected by photoevaporation of the gas disk as the giant migrates. The effect of this on terrestrial planet formation during and after giant planet migration will be examined in a future paper.

(iii) Type I migration. We have not included the effect of type I migration (Ward 1997; Tanaka et al. 2002; Tanaka \& Ward 2004), which would operate most effectively on sub-gap opening bodies of $\gtrsim 1 m_{\oplus}$, in any of our published models to date and have assumed that it does not play a major role. However, we discussed in detail its possible influence in Paper I and confine ourselves here to the further comment that even if type I migration operates with a reduced efficiency to that expected from the canonical model, which seems likely, it might still have a significant effect because of the sensitivity of our model's outcome to the dissipation exerted on solid bodies. Type I interactions would exert damping forces on protoplanets additional to dynamical friction. This could have the effect of reducing the eccentricity excitation of protoplanets captured at resonances with the giant planet, hence reducing their tendency to intersect the orbit of the giant and be scattered into the external disk. The overall effect might be to increase the shepherding effect of the giant planet at the expense of scattering, enhancing the fraction of surviving material found interior to the giant planet at the end of the simulation. If this material avoids migration into the central star, it is possible that inclusion of type I migration forces will assist in the formation and survival of hot-Neptune and/or hot-Earth systems. We are currently running simulations to examine this possibility.

(iv) Planetesimal size evolution. For computational simplicity we have assumed a uniform planetesimal population with radii of $10 \mathrm{~km}$. In reality there would be a distribution of planetesimal sizes determined from a balance between their rates of accumulation and fragmentation. In regions of the disk that are dynamically cold, the mean planetesimal size would grow via binary mergers, whilst in regions that are more dynamically excited destructive collisions could result in the planetesimal population being ground down into smaller fragments. Fragmentation of the planetesimal population in the context of the oligarchic growth regime however is not necessarily an obstacle to planet formation and may actually assist planetary growth by supplying protoplanets with a more strongly damped feedstock, enhancing the effect of gravitational focussing (Chambers 2006). A particularly challenging environment for planetesimal survival in our simulations in in the compacted region of the disk between the 4:3 and 2:1 resonances with the giant planet. In this region, planetesimal surface densities are enhanced (see the left hand panel in Fig. 8) and the population as a whole is strongly stirred generating eccentricities as high as $0.2-0.3$ (see Figs. 5 and 6). Random velocities of several $\mathrm{km}$ per second, far in excess of planetesimal escape velocities, are indicated and mutual planetesimal collisions would result in fragmentation. What this means for our model is unclear, but it is possible that the fragment population, which would be much more strongly affected by gas drag, would evolve inward rapidly, thereby escaping the dynamically excited zone. It could then be gathered efficiently by protoplanets and accreted. We speculate that the overall effect of this process might be to reduce the planetesimal fraction of the external disk material, but not to reduce its overall mass as protoplanets should be scattered there with the same, or even greater, efficiency.

(v) Eccentric giant planet. In this paper we have assumed that the giant planet is on a high circular orbit as it migrates. Nagasawa et al. (2005) have shown that the interior disk can be pre-stirred through the action of a sweeping secular resonance if the giant is on a modestly eccentric orbit. This would modify the accretion history of the inner disk.

\section{Conclusions}

In this paper we have presented the results of simulations that model terrestrial planet formation during and after the migration of a gas-giant planet to form a "hot-Jupiter". This work is an extension of our previous work (Fogg \& Nelson 2005), with improvements being made by modelling the viscous evolution of the gas disk, gap-formation and inner cavity formation in the gas disk due to the gravitational influence of the planet, and self-consistent type II migration. A popular belief has been that hot-Jupiter systems are unlikely to host terrestrial planets, as migration of the giant planet through the terrestrial planet zone 
was expected to sweep that region of planet forming material. We find, however, that the majority of this mass survives the migration episode as an interior or exterior disk remnant from which terrestrial planet formation can resume. This occurs via a combination of shepherding of the original solids disk ahead of the giant, and random scattering of the majority of this compacted material into orbits external to the giant. The net effect is not a disappearance of planetary building blocks from the inner system, but rather a stirring and mixing of material originally formed at different radial distances.

Now that more realistic gas dynamics have been included in our model, generating partial cavity formation close to the central star, gap formation in the vicinity of the giant planet's orbit, consistent type II migration rates, and a decline in the overall mass of gas present with time, we have found the following qualitative differences between the results presented here and in our previous work:

1. Scattering is favored over shepherding irrespective of the maturity of the inner solids disk at the epoch of giant planet migration. This occurs because of the rapid loss of gas from the disk interior to the giant, and in the vicinity of the giant planet's orbit, which reduces the damping of planetesimal trajectories. The efficiency of shepherding and dynamical friction is therefore reduced, increasing the probability that a given body will come close enough to the giant to be scattered into an external orbit. In all our scenarios $\gtrsim 60 \%$ of the original solids disk material survives in a regenerated external disk.

2. The principal mass loss mechanism is accretion by the giant planet, rather than the central star. This is because gas densities close to the central star are reduced by $2-3$ orders of magnitude, suppressing the gas-drag-induced orbital decay of planetesimals and allowing the giant planet to catch up with and sweep through even the innermost solids disk material. Most accretion by the giant occurs at late times when scattering by the giant becomes less effective due to the system being contained deep within the star's gravitational potential well.

3. Hot-Neptune and/or hot-Earth survival is not favored in our new model because of the enhanced tendency of the giant planet to scatter material outward and to accrete material at late times during the migration. Accelerated protoplanetary accretion within the compacted shepherded portion of the disk is observed, with objects growing to several $m_{\oplus}$, but their orbits typically destabilize at late times due to capture in eccentricity-pumping mean motion resonances, resulting in eventual collision with the giant planet. However, due to the sensitivity of our results to levels of dissipation close to the central star at late times, and the potential influence of type I migration and eccentricity damping that we have neglected, it is premature to rule out the possibility that hot-Neptunes or hot-Earths can form and survive interior to Jovian planets during their migration to become hot-Jupiters.

4. Whilst we predicted terrestrial planet formation in hotJupiter systems from the results of our previous model, the external disks generated by our new model appear to be even more benign places for this to occur. These disks contain a greater mass of material, are less dispersed, and are composed of a higher fraction of small bodies capable of exerting dynamical friction and hence damping the excited orbits of scattered protoplanets. The formation of terrestrial planets of masses in the range $1 \leq m_{\mathrm{p}} \leq 3 m_{\oplus}$ occurred in or near the habitable zone during a simulation that we continued after the giant stopped migrating. The radial mixing of volatilerich material from beyond the snowline means that terrestrial planets forming in the habitable zones of hot-Jupiter systems are likely to be "water-worlds", hosting deep, global oceans.

The results presented in this paper make a clear prediction that terrestrial planets will eventually be discovered in the habitable zones of hot-Jupiter systems. Such systems may be detectable by forthcoming missions such as KEPLER, DARWIN and TPF.

\section{References}

Alibert, Y., Mordasini, C., Benz, W., \& Winisdoerffer, C. 2005, A\&A, 434, 343 Artymowicz, P. 1993, ApJ, 419, 166

Armitage, P. J. 2003, ApJ, 582, L47

Armitage, P. J., Livio, M., Lubow, S. H., \& Pringle, J. E. 2002, MNRAS, 334, 248

Asphaug, E., Agnor, C. B., \& Williams, Q. 2006, Nature, 439, 155

Bonfils, X., Forveille, T., Delfosse, X., et al. 2005, A\&A, 443, L15

Bryden, G., Chen, X., Lin, D. N. C., et al. 1999, ApJ, 514, 344

Butler, P., Vogt, S., Marcy, G. W., et al. 2004, ApJ, 617, L580

Butler, P., Wright, J. T., Marcy, G. W., et al. 2006, ApJ, 646, 505

Canup, R. M., \& Pierazzo, E. 2006, LPI, 37, 2146

Chambers, J. E. 1999, MNRAS, 304, 793

Chambers, J. E. 2001, Icarus, 152, 205

Chambers, J. E. 2006, Icarus, 180, 496

Drake, M. J., \& Righter, K. 2002, Nature, 416, 39

Fischer, D. A., \& Valenti, J. 2005, ApJ, 622, 1102

Fogg, M. J., \& Nelson, R. P. 2005, A\&A, 441, 791

Goldreich, P., \& Soter, S. 1966, Icarus, 5, 375

Haisch, K. E., Lada, E. A., \& Lada, C. J. 2001, ApJ, 553, L153

Halliday, A. N. 2004, Nature, 427, 505

Hayashi, C. 1981, Prog. Theor. Phys. Suppl., 70, 35

Kasting, J. F., Whitmire, D. P., \& Reynolds, R. T. 1993, Icarus, 101, 108

Kleine, T., Münker, C., Mezger, K., \& Palme, H. 2002, Nature, 418, 952

Kokubo, E., \& Ida, S. 2000, Icarus 143, 15

Kuchner, M. J. 2003, ApJ, 596, L105

Léger, A., Selsis, F., Sotin, C., et al. 2004, Icarus, 169, 499

Lin, D. N. C., \& Papaloizou, J. C. B. 1986, ApJ, 309, 846

Lin, D. N. C., Bodenheimer, P., \& Richardson, D. C. 1996, Nature, 380, 606

Lineweaver, C. H. 2001, Icarus, 151, 307

Lineweaver, C. H., Fenner, Y., \& Gibson, B. K. 2004, Science, 303, 59

Lissauer, J. J. 1987, Icarus, 69, 249

Mandell, A. M., \& Sigurdsson, S. 2003, ApJ, 599, L111

McArthur, B. E., Endl, M., Cochran, G., et al. 2004, ApJ, 614, L81

Morbidelli, A., Chambers, J., Lunine, J. I., et al. 2000, Meteorit. Planet. Sci., 35, 1309

Nagasawa, M., Lin, D. N. C., \& Thommes, E. 2005, ApJ, 635, 578

Nelson, R. P., Papaloizou, J. C. B., Masset, F. S., \& Kley, W. 2000, MNRAS, 318, 18

Papaloizou, J. C. B., \& Nelson, R. P. 2005, A\&A, 433, 247

Pringle, J. E. 1981, ARA\&A, 19, 137

Raymond, S. N., Quinn, T., \& Lunine, J. I. 2004, Icarus, 168, 1

Raymond, S. N., Quinn, T., \& Lunine, J. I. 2005a, Icarus, 177, 256

Raymond, S. N., Quinn, T., \& Lunine, J. I. 2005b, Astrobiology, submitted [arXiv: astro-ph/0510285]

Raymond, S. N., Mandell, A. M., \& Sigurdsson, S. 2006, Science, 313, 1413

Rivera, E. J., Lissauer, J. J., Butler, R. P., et al. 2005, ApJ, 634, 625

Santos, N. C., Israelian, G., \& Mayor, M. 2004a, A\&A, 415,1153

Santos, N. C., Bouchy, F., Mayor, M., et al. 2004b, A\&A, 426, L19

Takeuchi, T., Miyama, S. N., \& Lin, D. N. C. 1996, ApJ, 460, 832

Tanaka, H., \& Ida, S. 1999, Icarus, 139, 366

Tanaka, H., \& Ward, W. R. 2004, ApJ, 602, 388

Tanaka, H., Takeuchi, T., \& Ward, W. R. 2002, ApJ, 565, 1257

Thommes, E. W., Duncan, M. J., \& Levison, H. F. 2003, Icarus, 161, 431

Trilling, D. E., Benz, W., Guillot, T., et al. 1998, ApJ, 500, 428

Vogt, S. S., Butler, R. P., Marcy, G. W., et al. 2005, ApJ, 632, 638

Udry, S., Mayor, M., Benz, W., et al. 2006, A\&A, 447, 361

Ward, P. D., \& Brownlee, D. 2000, Rare Earth: Why complex life is uncommon in the universe, (New York: Copernicus Books)

Ward, W. R. 1997, Icarus, 126, 261

Zhou, J. -L., Aarseth, S. J., Lin, D. N. C., \& Nagasawa, M. 2005, ApJ, 631, L85 